\title{
INFINITE-DIMENSIONAL INTEGRATION ON WEIGHTED HILBERT SPACES
}

\author{
MICHAEL GNEWUCH
}

\begin{abstract}
We study the numerical integration problem for functions with infinitely many variables which stem from a weighted reproducing kernel Hilbert space. We study the worst case $\varepsilon$-complexity which is defined as the minimal cost among all algorithms whose worst case error over the Hilbert space unit ball is at most $\varepsilon$. Here we assume that the cost of evaluating a function depends polynomially on the number of active variables. The infinite-dimensional integration problem is tractable if the $\varepsilon$-complexity is bounded by a constant times a power of $1 / \varepsilon$. The smallest such power is called the exponent of tractability.

We provide improved lower bounds for the exponent of tractability for general finite-order weights and, with the help of multilevel algorithms, improved upper bounds for three newly defined classes of finite-order weights. The newly defined finite-intersection weights model the situation where each group of variables interacts with at most $\rho$ other groups of variables, $\rho$ some fixed number. For these weights we obtain sharp upper bounds for any decay of the weights and any polynomial degree of the cost function. For the other two classes of finite-order weights our upper bounds are sharp if, e.g., the decay of the weights is fast or slow enough.

Furthermore, we deduce a lower bound for the exponent of tractability for arbitrary weights and a constructive upper bound for product weights.
\end{abstract}

\section{INTRODUCTION}

Integrals over functions with an unbounded or infinite number of variables are important in quantum chemistry and physics, as well as in financial mathematics; see, e.g., [8, 39] and the references mentioned therein. In this paper we study the numerical integration problem for functions defined over the infinite-dimensional unit cube $[0,1]^{\mathbb{N}}$. The functions belong to a reproducing kernel Hilbert space $\mathcal{H}_{\boldsymbol{\gamma}}$. Its kernel is built up from weighted sums of products of the 1-dimensional reproducing kernel $K(x, y)=\min \{x, y\}$. The role of the weights $\gamma$ is to moderate the importance of different groups of variables. In fact, the infinite-dimensional integration problem consists of infinitely many finite-dimensional integration sub-problems of varying importance, and the importance of each sub-problem is proportional to the corresponding weight. The finite-dimensional integration sub-problems are intimately related to $L_{2}$-star discrepancy.

We assume that the evaluation of functions from $\mathcal{H}_{\gamma}$ is only possible at points with finitely many components different from zero. (We call these components "active variables".) Furthermore, we assume that the cost of a single evaluation depends polynomially on the number of components different from zero.

Received by the editor May 21, 2010 and, in revised form, May 31, 2011.

2010 Mathematics Subject Classification. Primary 65C05, 65D30; Secondary 11K38.

(C) 2012 American Mathematical Society 
We study the worst case $\varepsilon$-complexity which is defined as the minimal cost among all algorithms whose worst case error over the Hilbert space unit ball is at most $\varepsilon$. The infinite-dimensional integration problem is said to be (polynomially) tractable if the $\varepsilon$-complexity is bounded by a constant times a power of $1 / \varepsilon$. The smallest such power is called the exponent of tractability.

Tractability of numerical integration for functions with an infinite number of variables has been studied, e.g., in [3, 18, 20, 22, 24, 28, 29, 34, 39]. Except for [20, 34, 39] all of these papers consider varying cost of function evaluations.

In this paper we study the setting proposed in [22]. In particular, we improve upper and lower bounds for the exponent of tractability provided in 22 for finiteorder weights, and improve on the upper bound given in 22 for product weights.

Let us explain our results in more detail. After describing the setting in Section 2 . we study finite-order weights in Section 3 . For finite-order weights $\gamma$ of order $\omega$, each function from the Hilbert space $\mathcal{H}_{\gamma}$ can be represented as a (usually infinite) sum of functions that depend on at most $\omega$ variables. Upper and lower bounds on the exponent of tractability have been provided in [22, Sect. 4]. The upper bound from [22] was achieved by employing an algorithm that uses only function evaluations at points with at most $\omega$ non-zero components.

In Section 3.1, we prove some lower bounds for the exponent of tractability. In Theorem 3.2 we show that if we restrict the class of admissible algorithms to those that use for fixed $B \geq \omega$ only function evaluations at points with at most $B$ non-zero components, then the upper bound on the exponent of tractability provided in [22, Sect. 4] is indeed sharp. We additionally introduce "cut-off weights" $\gamma^{(\sigma)}$ and quantities $t_{\sigma}^{*}, \sigma=1,2, \ldots$, which allow us a more careful analysis of the dependence of the infinite-dimensional integration problem on the given weights $\gamma$. This analysis enables us to improve the lower bound on the tractability exponent for general algorithms from [22, Sect. 4]; see Theorem 3.4. Afterwards we illustrate our lower bounds for three newly defined classes of finite order weights:

- finite-intersection weights (which, in particular, include finite-diameter weights),

- finite-product weights (which are defined as the product weights introduced by Sloan and Woźniakowski in [36], except that all weights of sets of variables with cardinality larger than some fixed $\omega$ are set to zero),

- lexicographically-ordered weights (whose properties complement the properties of the other two classes of weights).

In Section 3.2, we introduce an algorithm for the infinite-dimensional integration problem. It is especially useful in the case where the polynomial degree $s$ of the cost function is less than the order $\omega$ of the finite-order weights. As we will explain later, this case is indeed the most important one. The algorithm combines a multilevel idea with quasi-Monte Carlo integration using sample points whose projections onto important sets of coordinates exhibit a small $L_{2}$-star discrepancy. Multilevel algorithms for numerical integration have been introduced by Heinrich [15, 16] and Giles [8, 9]. Furthermore, multilevel algorithms have been used for infinitedimensional integration in [3, 10, 18, 24, 29]. For further references to multilevel ideas see the literature mentioned in these papers.

We use our algorithm to improve the upper bound on the tractability exponent from 22 for the three classes of finite-order weights mentioned above. 
In the case of finite-intersection weights our upper bound matches our lower bound for all values of $s$ and any decay of the weights; see Theorem 3.12. This is the first class of weights for which the exact exponent of tractability is known for any possible decay of the weights and for any polynomial degree of the cost function. The result relies, in particular, on the discrepancy result proved in Proposition 3.11 .

Our upper bounds for lexicographically-ordered and finite-product weights match the corresponding lower bounds if, e.g., the decay of the weights is fast or slow enough; see Theorem 3.14 and 3.16 .

In Section 4, we apply our methods and results for finite-order weights to the case of arbitrary weights. In Section 4.1, we state a new lower bound for the exponent of tractability for arbitrary weights, which is a direct corollary of Theorem 3.4. In particular, it generalizes the non-trivial lower bound for product weights that was proved in 22].

As already mentioned, our multilevel algorithm is especially useful for finite-order weights if $s<\omega$. For weights that are not of finite order, we have formally $\omega=\infty$; we call such weights "infinite-order weights". Therefore it is not too surprising that our algorithm should also lead to good results for infinite-order weights. We illustrate this for product weights in Theorem 4.2. The upper bound provided there improves significantly on the bounds provided in [22, Sect. 3]. In particular, we show that the exponent of tractability $p^{*}$ takes the optimal value $p^{*}=1$ if the decay of the weights is sufficiently fast. In contrast, if $s>0$ and the weights do not decay super-polynomially, the upper bound on $p^{*}$ from [22, Sect. 3] is strictly larger than 1. Moreover, our upper bound also matches the lower bound in the case where $s \leq 1$ and the decay of the weights is sufficiently slow. Independently from our work, the same result has been proved in the recent paper [29] by a different analysis for an alternative cost model, the so-called variable subspace sampling model. We will discuss this cost model and the result from [29] in detail in Remark 4.4 .

Between the submission and the revision of this article, two new papers on infinite-dimensional integration were completed [2, 33]. Both papers treat the randomized setting, and 33 in addition also the worst case (deterministic) setting. We added a brief discussion of the worst case setting results from [33] in Remark 4.5 .

Although we confine ourselves for the sake of clarity to explicit upper bounds for four classes of weights, we stress that our multilevel algorithm together with our generic (weight-specific) choice of quasi-Monte Carlo points from Proposition 3.9 is applicable to any class of weights.

Our results can be extended to more general reproducing kernel Hilbert spaces. Since this paper is already rather long, we do not discuss these kinds of generalizations in detail. Natural function space settings to which our analysis and our algorithm can be generalized are, e.g., considered in [22, Sect. 5] and [29].

\section{The SETTING}

In this paper, we consider the setting studied in 22. Let us recall the basic notions from [22, Sect. 2] and add some definitions and notation that are helpful to describe our results.

Let us start with some general notation: For $d \in \mathbb{N}$ we denote by $[d]$ the set $\{1,2, \ldots, d\}$. Furthermore, we denote the cardinality of a finite set $A$ by $|A|$. 
2.1. Weights. Let $\gamma=\left\{\gamma_{\mathfrak{u}}\right\}_{\mathfrak{u} \subset \mathbb{N} ;|\mathfrak{u}|<\infty}$ be a given set of non-negative numbers $\gamma_{\mathfrak{u}}$ that are called weights. For a given set of weights $\gamma$ we denote by $\widehat{\gamma}$ the set of weights defined by

$$
\widehat{\gamma}_{\mathfrak{u}}:=\gamma_{\mathfrak{u}} 3^{-|\mathfrak{u}|} \text { for all finite } \mathfrak{u} \subset \mathbb{N} \text {. }
$$

Weights $\gamma$ are called finite-order weights of order $\omega$ if there exists an $\omega \in \mathbb{N}$ such that $\gamma_{\mathfrak{u}}=0$ for all $\mathfrak{u} \in \mathbb{N}$ with $|\mathfrak{u}|>\omega$. Finite-order weights were introduced by Dick et al. in 5 for spaces of functions with a finite number of variables.

The weights we introduce in the following definition will be essential for our analysis.

Definition 2.1. For weights $\gamma$ and $\sigma \in \mathbb{N}$ let us define the cut-off weights of order $\sigma$ :

$$
\gamma^{(\sigma)}=\left\{\gamma_{\mathfrak{u}}^{(\sigma)}\right\}_{\mathfrak{u} \subset \mathbb{N} ;|\mathfrak{u}|<\infty} \quad \text { via } \quad \gamma_{\mathfrak{u}}^{(\sigma)}= \begin{cases}\gamma_{\mathfrak{u}} & \text { if }|\mathfrak{u}| \leq \sigma \\ 0 & \text { otherwise. }\end{cases}
$$

Clearly, cut-off weights of order $\sigma$ are in particular finite-order weights of order $\sigma$.

Let us now assume that we have a bounded set of weights $\left\{\gamma_{\mathfrak{u}}^{(\sigma)}\right\}_{\mathfrak{u} \subset \mathbb{N} ;|\mathfrak{u}|<\infty}$ that has 0 as the sole accumulation point. Note that the set $\{\mathfrak{u} \subset \mathbb{N}|| \mathfrak{u} \mid<\infty\}$ is infinite, but still countable. Thus let us denote by $\mathfrak{u}_{1}(\sigma), \mathfrak{u}_{2}(\sigma), \ldots$, the non-empty sets $\mathfrak{u} \subset \mathbb{N}$ with $\gamma_{\mathfrak{u}}^{(\sigma)}>0$ for which $\widehat{\gamma}_{\mathfrak{u}_{1}(\sigma)}^{(\sigma)} \geq \widehat{\gamma}_{\mathfrak{u}_{2}(\sigma)}^{(\sigma)} \geq \cdots$. Let us put $\mathfrak{u}_{0}(\sigma):=\emptyset$. We can make the same definitions for $\sigma=\infty$; then we have obviously $\gamma^{(\infty)}=\gamma$. For convenience we will usually suppress any reference to $\sigma$ in the case where $\sigma=\infty$. For $\sigma \in \mathbb{N} \cup\{\infty\}$ let us define

$$
\operatorname{tail}_{\boldsymbol{\gamma}, \sigma}(d):=\sum_{j=d+1}^{\infty} \widehat{\gamma}_{\mathfrak{u}_{j}(\sigma)}^{(\sigma)} \in[0, \infty]
$$

and

$$
\operatorname{decay}_{\boldsymbol{\gamma}, \sigma}:=\sup \left\{p \in \mathbb{R} \mid \lim _{j \rightarrow \infty} \widehat{\gamma}_{\mathfrak{u}_{j}(\sigma)}^{(\sigma)} j^{p}=0\right\} .
$$

Next we define quantities that describe for $\sigma \in \mathbb{N} \cup\{\infty\}$, roughly speaking, the density of the set system $\left\{\mathfrak{u}_{1}(\sigma), \mathfrak{u}_{2}(\sigma), \ldots\right\}$ in $\{\mathfrak{v} \subset \mathbb{N}|| \mathfrak{v} \mid<\infty\}$. As we shall see later, these quantities provide essentially an upper bound on the efficiency of projection, i.e., on the number of non-trivial finite-dimensional integration subproblems we can tackle by using sample points with active variables in coordinate sets $\mathfrak{v} \subset \mathbb{N},|\mathfrak{v}|<\infty$.

Definition 2.2. For $\sigma \in \mathbb{N} \cup\{\infty\}$ let $t_{\sigma}^{*} \in[0, \infty]$ be defined as

$$
t_{\sigma}^{*}=\inf \left\{t \geq\left. 0\left|\exists C_{t}>0 \forall \mathfrak{v} \subseteq \mathbb{N}:\right| \mathfrak{v}|<\infty \Longrightarrow|\left\{i \in \mathbb{N} \mid \mathfrak{u}_{i}(\sigma) \subseteq \mathfrak{v}\right\}\left|\leq C_{t}\right| \mathfrak{v}\right|^{t}\right\} .
$$

Let $\sigma \in \mathbb{N}$. Since $\left|\mathfrak{u}_{i}(\sigma)\right| \leq \sigma$ for all $i \in \mathbb{N}$, we have obviously $t_{\sigma}^{*} \leq \sigma$. On the other hand, if we have an infinite sequence $\left\{\mathfrak{u}_{j}(\sigma)\right\}_{j \in \mathbb{N}}$, it is easy to see that $t_{\sigma}^{*} \geq 1$. Indeed, define $V_{k}=\bigcup_{i=1}^{k} \mathfrak{u}_{i}(\sigma)$. Then $k \leq\left|\left\{i \in \mathbb{N} \mid \mathfrak{u}_{i}(\sigma) \subseteq V_{k}\right\}\right|$ and $\left|V_{k}\right| \leq k \sigma$. Thus, if there exist $t, C_{t}>0$ such that $\left|\left\{i \in \mathbb{N} \mid \mathfrak{u}_{i}(\sigma) \subseteq V_{k}\right\}\right| \leq C_{t}\left|V_{k}\right|^{t}$ for all $k \in \mathbb{N}$, then necessarily $C_{t} \geq k^{1-t} \sigma^{-t}$ for all $k$. Hence $t \geq 1$. 
2.2. Weighted Hilbert spaces. In this subsection, we define the weighted reproducing kernel Hilbert spaces whose functions serve as our integrands. Our standard reference for basic properties of reproducing kernel Hilbert spaces and their kernels is [1. Additional information about the reproducing kernel Hilbert spaces we consider here can, e.g., be found in [20, 22].

Let

$$
K:[0,1] \times[0,1] \rightarrow \mathbb{R} \quad \text { be given by } \quad K(x, y):=\min \{x, y\} .
$$

$K$ is a reproducing kernel, and the corresponding reproducing kernel Hilbert space $H(K)$ is the space of absolutely continuous functions $f:[0,1] \rightarrow \mathbb{R}$ with $f(0)=0$, whose weak derivatives $f^{\prime}$ are in $L_{2}([0,1])$, the space of square-integrable functions on $[0,1]$. Its inner product is given by

$$
\langle f, g\rangle_{H(K)}=\int_{0}^{1} f^{\prime}(x) g^{\prime}(x) \mathrm{d} x,
$$

and the reproducing property reads as

$$
f(x)=\langle f, K(x, \cdot)\rangle_{H(K)} \text { for all } f \in H(K), x \in[0,1] .
$$

Let $\gamma=\left\{\gamma_{\mathfrak{u}}\right\}_{\mathfrak{u} \subset \mathbb{N} ;|\mathfrak{u}|<\infty}$ be a given set of weights. If not explicitly stated otherwise, we always require that

$$
\gamma_{\emptyset}=1 \quad \text { and } \sum_{\mathfrak{u} \subset \mathbb{N} ;|\mathfrak{u}|<\infty} \gamma_{\mathfrak{u}}<\infty,
$$

and assume furthermore that for at least one finite, non-empty subset $\mathfrak{u}$ of $\mathbb{N}$ we have $\gamma_{\mathfrak{u}}>0$. Note that (3) implies that decay ${ }_{\boldsymbol{\gamma}, \sigma} \geq 1$ for all $\sigma \in \mathbb{N} \cup\{\infty\}$. For infinite-dimensional vectors $\boldsymbol{x}, \boldsymbol{y} \in[0,1]^{\mathbb{N}}$, define

$$
\mathcal{K}_{\gamma}(\boldsymbol{x}, \boldsymbol{y}):=\sum_{\mathfrak{u} \subset \mathbb{N} ;|\mathfrak{u}|<\infty} \gamma_{\mathfrak{u}} K_{\mathfrak{u}}(\boldsymbol{x}, \boldsymbol{y})
$$

where

$$
K_{\mathfrak{u}}(\boldsymbol{x}, \boldsymbol{y}):=\prod_{j \in \mathfrak{u}} K\left(x_{j}, y_{j}\right)=\prod_{j \in \mathfrak{u}} \min \left\{x_{j}, y_{j}\right\} .
$$

Here we use the convention that the empty product is 1 . Since the function $K$ takes only values in $[0,1]$, condition (3) implies that $\mathcal{K}_{\gamma}$ is pointwise well-defined and bounded. It is well known that $K_{\mathfrak{u}}$ and $\mathcal{K}_{\gamma}$ are also reproducing kernels; see [1]. The reproducing kernel Hilbert space $\mathcal{H}_{\gamma}:=H\left(\mathcal{K}_{\gamma}\right)$ corresponding to $\mathcal{K}_{\gamma}$ consists of functions $f, g:[0,1]^{\mathbb{N}} \rightarrow \mathbb{R}$ which are once weakly differentiable with respect to all variables, and their mixed weak derivatives are square integrable. Its inner product is given by

$(5)\langle f, g\rangle_{\mathcal{H}_{\gamma}}=f(\mathbf{0}) g(\mathbf{0})+\sum_{\mathfrak{u} \subset \mathbb{N} ; 1 \leq|\mathfrak{u}|<\infty} \frac{1}{\gamma_{\mathfrak{u}}} \int_{[0,1]|\mathfrak{u}|} \frac{\partial^{|\mathfrak{u}|}}{\partial \boldsymbol{x}_{\mathfrak{u}}} f\left(\boldsymbol{x}_{\mathfrak{u}} ; \mathbf{0}\right) \frac{\partial^{|\mathfrak{u}|}}{\partial \boldsymbol{x}_{\mathfrak{u}}} g\left(\boldsymbol{x}_{\mathfrak{u}} ; \mathbf{0}\right) \mathrm{d} \boldsymbol{x}_{\mathfrak{u}}$,

where we use the convention $0 / 0=0$. Here, $\boldsymbol{x}_{\mathfrak{u}}=\left(x_{j}\right)_{j \in \mathfrak{u}}$ is a vector with $|\mathfrak{u}|$ components and $\left(\boldsymbol{x}_{\mathfrak{u}} ; \mathbf{0}\right)$ denotes the vector $\boldsymbol{y}=\left(y_{1}, y_{2}, \ldots\right) \in[0,1]^{\mathbb{N}}$ with $y_{j}=x_{j}$ if $j \in \mathfrak{u}$ and $y_{j}=0$ otherwise. Furthermore, $\partial^{|\mathfrak{u}|} / \partial \boldsymbol{x}_{\mathfrak{u}}$ is a simplified notation for $\prod_{j \in \mathfrak{u}}\left(\partial / \partial x_{j}\right)$. We denote the norm in $\mathcal{H}_{\boldsymbol{\gamma}}$ by $\|\cdot\|_{\mathcal{H}_{\boldsymbol{\gamma}}}$. If $\gamma_{\mathfrak{u}}=0$, then we have for all $f \in \mathcal{H}_{\gamma}$,

$$
\frac{\partial^{|\mathfrak{u}|}}{\partial \boldsymbol{x}_{\mathfrak{u}}} f\left(\boldsymbol{x}_{\mathfrak{u}} ; \mathbf{0}\right)=0 \quad \text { for almost all } \quad \boldsymbol{x}_{\mathfrak{u}} \in[0,1]^{|\mathfrak{u}|} .
$$


For a finite subset $\mathfrak{u} \subset \mathbb{N}$, let $H\left(K_{\mathfrak{u}}\right)$ denote the Hilbert space with reproducing kernel $K_{\mathfrak{u}}$. For $\mathfrak{u}=\emptyset$ we have $H\left(K_{\emptyset}\right)=\operatorname{span}\{1\}$, where 1 is the constant function taking only the value 1 . For non-empty $\mathfrak{u}$, a function in $H\left(K_{\mathfrak{u}}\right)$ depends only on the variables $x_{j}, j \in \mathfrak{u}$, and vanishes whenever $x_{j}=0$ for some $j \in \mathfrak{u}$. For $\mathfrak{u} \neq \emptyset$, the inner product in $H\left(K_{\mathfrak{u}}\right)$ is

$$
\langle f, g\rangle_{H\left(K_{\mathfrak{u}}\right)}=\int_{[0,1]^{|\mathfrak{u}|}} \frac{\partial^{|\mathfrak{u}|}}{\partial \boldsymbol{x}_{\mathfrak{u}}} f\left(\boldsymbol{x}_{\mathfrak{u}} ; \mathbf{0}\right) \frac{\partial^{|\mathfrak{u}|}}{\partial \boldsymbol{x}_{\mathfrak{u}}} g\left(\boldsymbol{x}_{\mathfrak{u}} ; \mathbf{0}\right) \mathrm{d} \boldsymbol{x}_{\mathfrak{u}},
$$

implying

$$
\|f\|_{\mathcal{H}_{\gamma}}=\gamma_{\mathfrak{u}}^{-1 / 2}\|f\|_{H\left(K_{\mathfrak{u}}\right)} \text { for all } f \in H\left(K_{\mathfrak{u}}\right) .
$$

For $\mathfrak{u} \neq \mathfrak{v}$ the spaces $H\left(K_{\mathfrak{u}}\right)$ and $H\left(K_{\mathfrak{v}}\right)$ are orthogonal, i.e., the space $\mathcal{H}_{\boldsymbol{\gamma}}$ is the direct orthogonal sum

$$
\mathcal{H}_{\gamma}=\bigoplus_{\mathfrak{u} \subset \mathbb{N} ;|\mathfrak{u}|<\infty} H\left(K_{\mathfrak{u}}\right)
$$

For finite $\mathfrak{u} \subset \mathbb{N}$, let $P_{\mathfrak{u}}$ denote the orthogonal projection

$$
P_{\mathfrak{u}}: \mathcal{H}_{\gamma} \rightarrow H\left(K_{\mathfrak{u}}\right), f \mapsto f_{\mathfrak{u}} .
$$

Then any function $f \in \mathcal{H}_{\gamma}$ has the unique orthogonal representation

$$
f=\sum_{\mathfrak{u} \subset \mathbb{N} ;|\mathfrak{u}|<\infty} f_{\mathfrak{u}} \quad \text { with } \quad f_{\mathfrak{u}}=P_{\mathfrak{u}}(f) \in H\left(K_{\mathfrak{u}}\right) .
$$

This implies

$$
\|f\|_{\mathcal{H}_{\gamma}}^{2}=\sum_{\mathfrak{u} \subset \mathbb{N} ;|\mathfrak{u}|<\infty}\left\|f_{\mathfrak{u}}\right\|_{\mathcal{H}_{\gamma}}^{2}=\sum_{\mathfrak{u} \subset \mathbb{N} ;|\mathfrak{u}|<\infty} \gamma_{\mathfrak{u}}^{-1}\left\|f_{\mathfrak{u}}\right\|_{H\left(K_{\mathfrak{u}}\right)}^{2} .
$$

The decomposition in (8) is a special case of the projection decomposition discussed in 20] and the infinite-dimensional generalization of the anchored decomposition discussed in [23], with anchor at the origin. As already said, each function $f_{\mathfrak{u}}$ depends only on the variables with indices in $\mathfrak{u}$. To stress this fact we write, as in 22 ,

$$
f_{\mathfrak{u}}(\boldsymbol{x})=f_{\mathfrak{u}}\left(\boldsymbol{x}_{\mathfrak{u}} ; \mathbf{0}\right)=f_{\mathfrak{u}}\left(\boldsymbol{x}_{\mathfrak{u}}\right) ;
$$

here and in the rest of the paper we use the convention to denote for a vector $\boldsymbol{x} \in[0,1]^{\mathbb{N}}$ the $|\mathfrak{u}|$-dimensional vector $\left(x_{j}\right)_{j \in \mathfrak{u}}$ by $\boldsymbol{x}_{\mathfrak{u}}$.

2.3. Infinite-dimensional integrals. For $f \in \mathcal{H}_{\boldsymbol{\gamma}}$, we want to approximate the infinite-dimensional integral

$$
\mathcal{I}_{\infty}(f)=\int_{[0,1]^{\mathbb{N}}} f(\boldsymbol{x}) \mathrm{d} \boldsymbol{x},
$$

where $\mathrm{d} \boldsymbol{x}$ is the infinite product measure of the Lebesgue measure restricted to $[0,1]$. Since $f(\boldsymbol{x})=\left\langle f, \mathcal{K}_{\boldsymbol{\gamma}}(\boldsymbol{x}, \cdot)\right\rangle_{\mathcal{H}_{\gamma}}$ for all $f \in \mathcal{H}_{\boldsymbol{\gamma}}$ and all $\boldsymbol{x} \in[0,1]^{\mathbb{N}}$, we can represent $\mathcal{I}_{\infty}$ as

$$
\mathcal{I}_{\infty}(f)=\langle f, h\rangle_{\mathcal{H}_{\gamma}} \quad \text { for all } \quad f \in \mathcal{H}_{\gamma},
$$

where the representer $h \in \mathcal{H}_{\boldsymbol{\gamma}}$ is given by

$$
h(\boldsymbol{x})=\int_{[0,1]^{\mathbb{N}}} \mathcal{K}_{\boldsymbol{\gamma}}(\boldsymbol{x}, \boldsymbol{y}) \mathrm{d} \boldsymbol{y}=\sum_{\mathfrak{u} \subset \mathbb{N} ;|\mathfrak{u}|<\infty} \gamma_{\mathfrak{u}} \prod_{j \in \mathfrak{u}}\left(x_{j}-\frac{1}{2} x_{j}^{2}\right) .
$$


The operator norm of the functional $\mathcal{I}_{\infty}$ is given by

$$
\left\|\mathcal{I}_{\infty}\right\|_{\mathcal{H}_{\gamma}}=\|h\|_{\mathcal{H}_{\gamma}}=\left(\sum_{\mathfrak{u} \subset \mathbb{N} ;|\mathfrak{u}|<\infty} \widehat{\gamma}_{\mathfrak{u}}\right)^{1 / 2} .
$$

For finite $\mathfrak{u} \subset \mathbb{N}$ and $f \in \mathcal{H}_{\gamma}$, define $I_{\mathfrak{u}}:=\mathcal{I}_{\infty} \circ P_{\mathfrak{u}}$, i.e., $I_{\mathfrak{u}}(f)=\left\langle f, P_{\mathfrak{u}}(h)\right\rangle_{\mathcal{H}_{\boldsymbol{\gamma}}}$. Thus

$$
I_{\mathfrak{u}}(f)=\int_{[0,1]^{|\mathfrak{u}|}} f_{\mathfrak{u}}\left(\boldsymbol{x}_{\mathfrak{u}}\right) \mathrm{d} \boldsymbol{x}_{\mathfrak{u}},
$$

and the representer $h_{\mathfrak{u}}$ of $I_{\mathfrak{u}}$ is given by

$$
h_{\mathfrak{u}}\left(\boldsymbol{x}_{\mathfrak{u}}\right)=P_{\mathfrak{u}}(h)\left(\boldsymbol{x}_{\mathfrak{u}}\right)=\gamma_{\mathfrak{u}} \prod_{j \in \mathfrak{u}}\left(x_{j}-\frac{1}{2} x_{j}^{2}\right) .
$$

Using this notation, we get

$$
\mathcal{I}_{\infty}(f)=\sum_{\mathfrak{u} \subset \mathbb{N} ;|\mathfrak{u}|<\infty} I_{\mathfrak{u}}\left(f_{\mathfrak{u}}\right) \text { for all } f \in \mathcal{H}_{\gamma} .
$$

2.4. Algorithms. As in [22, we assume that we can compute $f(\boldsymbol{x})$ only for $\boldsymbol{x} \in$ $[0,1]^{\mathbb{N}}$ with finitely many components different from zero. We further assume that for each $f \in \mathcal{H}_{\boldsymbol{\gamma}}$, each finite $\mathfrak{u} \subset \mathbb{N}$, and each vector $\boldsymbol{x}_{\mathfrak{u}}$, whose components are all different from zero, the cost of computing $f\left(\boldsymbol{x}_{\mathfrak{u}} ; \mathbf{0}\right)$ is equal to $\$(|\mathfrak{u}|)$ for a given non-decreasing cost function

$$
\$: \mathbb{N}_{0} \rightarrow[1, \infty)
$$

where $\mathbb{N}_{0}:=\{0\} \cup \mathbb{N}$. For our lower bounds we will usually assume $\$(k)=\Omega\left(k^{s}\right)$ (i.e., $k^{s}=O(\$(k))$ ), for our upper bounds $\$(k)=O\left(k^{s}\right)$ for some non-negative $s$.

Since the problem of approximating $\mathcal{I}_{\infty}$ is linear and we want to study the worst case error of algorithms over a convex and balanced set, it is known; see, e.g., 38, that non-linear algorithms and adaption do not help. Due to this, we consider, without loss of generality, linear algorithms of the form

$$
\mathcal{Q}(f)=\sum_{i=1}^{n} a_{i} f\left(\boldsymbol{x}_{\mathfrak{v}_{i}}^{(i)} ; \mathbf{0}\right)
$$

for some $n \in \mathbb{N}$, finite sets $\mathfrak{v}_{i} \subset \mathbb{N}, a_{i} \in \mathbb{R}$, and sampling points $\left(\boldsymbol{x}_{\mathfrak{v}_{i}}^{(i)} ; \mathbf{0}\right)$ for $i=1,2, \ldots, n$, where we assume that all components of $\boldsymbol{x}_{\mathfrak{v}_{i}}^{(i)}$ are non-zero.

The cost of the algorithm $\mathcal{Q}$ is then defined by

$$
\operatorname{cost}(\mathcal{Q}):=\sum_{i=1}^{n} \$\left(\left|\mathfrak{v}_{i}\right|\right)
$$

The worst case error of $\mathcal{Q}$ is defined by

$$
e\left(\mathcal{Q} ; \mathcal{H}_{\gamma}\right):=\sup _{\|f\|_{\mathcal{H}_{\gamma} \leq 1}}\left|\mathcal{I}_{\infty}(f)-\mathcal{Q}(f)\right|=\left\|\mathcal{I}_{\infty}-\mathcal{Q}\right\|_{\mathcal{H}_{\gamma}} .
$$

We may express the approximation error as

$$
\mathcal{I}_{\infty}(f)-\mathcal{Q}(f)=\left\langle f, h-h_{\mathcal{Q}}\right\rangle_{\mathcal{H}_{\gamma}}, \quad \text { where } h_{\mathcal{Q}}:=\sum_{i=1}^{n} a_{i} \mathcal{K}_{\boldsymbol{\gamma}}\left(\left(\boldsymbol{x}_{\mathfrak{v}_{i}}^{(i)} ; \mathbf{0}\right), \cdot\right)
$$


This implies, in particular, $e\left(\mathcal{Q} ; \mathcal{H}_{\gamma}\right)=\left\|\mathcal{I}_{\infty}-\mathcal{Q}\right\|_{\mathcal{H}_{\gamma}}=\left\|h-h_{\mathcal{Q}}\right\|_{\mathcal{H}_{\gamma}}$. For a finite $\mathfrak{u} \subset \mathbb{N}$, define $\mathcal{Q}_{\mathfrak{u}}:=\mathcal{Q} \circ P_{\mathfrak{u}}$, i.e.,

$$
\mathcal{Q}_{\mathfrak{u}}(f)=\left\langle f, P_{\mathfrak{u}}\left(h_{\mathcal{Q}}\right)\right\rangle_{\mathcal{H}_{\gamma}}=\sum_{i: \mathfrak{u} \subseteq \mathfrak{v}_{i}} a_{i} f_{\mathfrak{u}}\left(\boldsymbol{x}_{\mathfrak{u}}^{(i)} ; \mathbf{0}\right) .
$$

Due to (44) and the uniqueness of the orthogonal representation (8), the representer $h_{\mathcal{Q}, \mathfrak{u}}$ of $\mathcal{Q}_{\mathfrak{u}}$ is given by

$$
h_{\mathcal{Q}, \mathfrak{u}}=P_{\mathfrak{u}}\left(h_{\mathcal{Q}}\right)=\sum_{i: \mathfrak{u} \subseteq \mathfrak{v}_{i}} a_{i} \gamma_{\mathfrak{u}} K_{\mathfrak{u}}\left(\left(\boldsymbol{x}_{\mathfrak{u}}^{(i)} ; \mathbf{0}\right), \cdot\right) .
$$

Using this notation, we get

$$
\mathcal{Q}(f)=\sum_{\mathfrak{u} \subset \mathbb{N} ;|\mathfrak{u}|<\infty} \mathcal{Q}_{\mathfrak{u}}\left(f_{\mathfrak{u}}\right)
$$

and the useful identity

$$
\left[e\left(\mathcal{Q} ; \mathcal{H}_{\gamma}\right)\right]^{2}=\sum_{\mathfrak{u} \subset \mathbb{N} ;|\mathfrak{u}|<\infty}\left\|I_{\mathfrak{u}}-\mathcal{Q}_{\mathfrak{u}}\right\|_{\mathcal{H}_{\gamma}}^{2}=\sum_{\mathfrak{u} \subset \mathbb{N} ;|\mathfrak{u}|<\infty} \gamma_{\mathfrak{u}}\left\|I_{\mathfrak{u}}-\mathcal{Q}_{\mathfrak{u}}\right\|_{H\left(K_{\mathfrak{u}}\right)}^{2} .
$$

Please note that (13) is just a mathematical identity and not a description of how the algorithm $Q$ is actually executed. If we speak about an algorithm $Q$ of form (10), then it will always be executed in its form (10). Consequently, its cost is always given by (11).

2.5. Discrepancy. High- and infinite-dimensional integration on reproducing kernel Hilbert spaces are intimately related to discrepancy; see, e.g., the papers [17, 36, 20, 30, 11] or the recent monograph [32]. In particular, for finite $\mathfrak{u} \subset \mathbb{N}$ the worst case error of multivariate numerical integration on $H\left(K_{\mathfrak{u}}\right)$ is related to the $L_{2}$-star discrepancy.

For a point set $T=\left\{\boldsymbol{t}^{(1)}, \ldots, \boldsymbol{t}^{(n)}\right\} \subset[0,1]^{|\mathfrak{u}|}$ and coefficients $\boldsymbol{a}=\left(a_{1}, \ldots, a_{n}\right) \in$ $\mathbb{R}^{n}$ let us define the $L_{2}$-star discrepancy of $T$ with respect to $\boldsymbol{a}$ by

$$
\operatorname{disc}_{2,|\mathfrak{u}|}^{*}(\boldsymbol{a}, T):=\left(\int_{[0,1]|\mathfrak{u}|}\left(\prod_{j \in \mathfrak{u}} x_{j}-\sum_{j=1}^{n} a_{j} 1_{\left[\mathbf{0}, \boldsymbol{x}_{\mathfrak{u}}\right)}\left(\boldsymbol{t}^{(j)}\right)\right)^{2} \mathrm{~d} \boldsymbol{x}_{\mathfrak{u}}\right)^{1 / 2},
$$

where $\left[\mathbf{0}, \boldsymbol{x}_{\mathfrak{u}}\right)=\prod_{j \in \mathfrak{u}}\left[0, x_{j}\right)$ and $1_{\left[\mathbf{0}, \boldsymbol{x}_{\mathfrak{u}}\right)}$ denotes the characteristic function of the set $\left[\mathbf{0}, \boldsymbol{x}_{\mathfrak{u}}\right)$. In the case where all coefficients are $a_{i}=1 / n$, we suppress the explicit reference to $\boldsymbol{a}$.

Let $\mathbf{1}:=(1,1, \ldots, 1) \in[0,1]^{|\mathfrak{u}|}$ and denote the points $\mathbf{1}-\boldsymbol{t}^{(j)}$ by $\overline{\boldsymbol{t}}^{(j)}$ and the set $\left\{\overline{\boldsymbol{t}}^{(1)}, \overline{\boldsymbol{t}}^{(2)}, \ldots, \overline{\boldsymbol{t}}^{(n)}\right\}$ by $\bar{T}$. If the linear algorithm $\widetilde{\mathcal{Q}}_{\mathfrak{u}}$ is given by

$$
\widetilde{\mathcal{Q}}_{\mathfrak{u}}\left(f_{\mathfrak{u}}\right)=\sum_{i=1}^{n} a_{i} f_{\mathfrak{u}}\left(\boldsymbol{t}^{(i)}\right) \quad \text { for } f_{\mathfrak{u}} \in H\left(K_{\mathfrak{u}}\right),
$$

then it is well known that the worst case error of approximating $I_{\mathfrak{u}}$ by $\tilde{\mathcal{Q}}_{\mathfrak{u}}$ is

$$
e\left(\widetilde{\mathcal{Q}}_{\mathfrak{u}}, H\left(K_{\mathfrak{u}}\right)\right):=\left\|I_{\mathfrak{u}}-\widetilde{\mathcal{Q}}_{\mathfrak{u}}\right\|_{H\left(K_{\mathfrak{u}}\right)}=\operatorname{disc}_{2,|\mathfrak{u}|}^{*}(\boldsymbol{a}, \bar{T}) ;
$$

see, e.g., [32, Ch. 9]. In particular, we have for the zero algorithm, i.e., the algorithm which approximates $I_{\mathfrak{u}}(f)$ for all $f$ by 0 ,

$$
e\left(\mathbf{0}, H\left(K_{\mathfrak{u}}\right)\right)=\left\|I_{\mathfrak{u}}\right\|_{H\left(K_{\mathfrak{u}}\right)}=3^{-|\mathfrak{u}| / 2} .
$$


Another important discrepancy measure is the star discrepancy of $T$ with respect to the set of coefficients $\boldsymbol{a}$, given by

$$
\operatorname{disc}_{\infty,|\mathfrak{u}|}^{*}(\boldsymbol{a}, T):=\sup _{\boldsymbol{x}_{\mathfrak{u}} \in[0,1]^{|\mathfrak{u}|}}\left|\prod_{j \in \mathfrak{u}} x_{j}-\sum_{j=1}^{n} a_{j} 1_{\left[\mathbf{0}, \boldsymbol{x}_{\mathfrak{u}}\right)}\left(\boldsymbol{t}^{(j)}\right)\right| .
$$

Obviously, we have always $\operatorname{disc}_{2,|\mathfrak{u}|}^{*}(\boldsymbol{a}, T) \leq \operatorname{disc}_{\infty,|\mathfrak{u}|}^{*}(\boldsymbol{a}, T)$. This is a useful relation, since the star discrepancy of projections of a point set is always at most as large as the star discrepancy of the point set itself and the construction of point sets with low star discrepancy has been intensively studied in the past; see, e.g., the papers [14, 37, 7, 25, 27, 21, 6], the monographs [26, 32], and the literature mentioned therein.

2.6. Polynomial tractability. The worst case $\varepsilon$-complexity is defined as the minimal cost among all algorithms of the form (10), whose worst case errors are at most $\varepsilon$, i.e.,

$$
\operatorname{comp}\left(\varepsilon ; \mathcal{H}_{\gamma}\right):=\inf \left\{\operatorname{cost}(\mathcal{Q}) \mid \mathcal{Q} \text { is of the form (10) and } e\left(\mathcal{Q} ; \mathcal{H}_{\gamma}\right) \leq \varepsilon\right\} .
$$

The integration problem $\mathcal{I}_{\infty}$ is said to be polynomially tractable if there are nonnegative constants $C$ and $p$ such that

$$
\operatorname{comp}\left(\varepsilon ; \mathcal{H}_{\gamma}\right) \leq C \varepsilon^{-p} \quad \text { for all } \varepsilon>0 .
$$

The exponent of polynomial tractability is given by

$$
p^{*}=p^{*}(\gamma):=\inf \{p \mid p \text { satisfies (18) }\} .
$$

If $\mathfrak{u} \neq \emptyset$, then it is well known that for the space $H\left(K_{\mathfrak{u}}\right)$ the minimal worst case error of algorithms using $n$ function values is of order at least $n^{-1}$. Since we assumed in Section 2.1 that at least one $\gamma_{\mathfrak{u}}$ is positive for some $\mathfrak{u} \neq \emptyset$, we have $\operatorname{comp}\left(\varepsilon ; \mathcal{H}_{\gamma}\right)=\Omega\left(\varepsilon^{-1}\right)$, and therefore $p^{*} \geq 1$. Essentially, $1 / p^{*}$ is the optimal convergence rate of the $N$ th minimal worst case error

$$
e_{\boldsymbol{\gamma}}(N):=\inf \left\{e\left(\mathcal{Q} ; \mathcal{H}_{\boldsymbol{\gamma}}\right) \mid \mathcal{Q} \text { is of the form (10) and } \operatorname{cost}(\mathcal{Q}) \leq N\right\} .
$$

One can, of course, also be interested in studying different notions of tractability. For multivariate problems such notions have been defined and studied; e.g., in [12, 13, 31. Here in this paper we focus solely on polynomial tractability. That is why we use from now on the shorthand "tractability" for "polynomial tractability".

\section{Finite-ORDER WEIGHTS}

Let us consider finite-order weights $\gamma=\left\{\gamma_{\mathfrak{u}}\right\}_{\mathfrak{u} \subset \mathbb{N} ;|\mathfrak{u}|<\infty}$ of order $\omega$. Due to our convention of notation, we have $\gamma=\gamma^{(\omega)}=\gamma^{(\infty)}$. Note that for finite-order weights of order $\omega$ we have $\widehat{\gamma}_{\mathfrak{u}} \leq \gamma_{\mathfrak{u}} \leq 3^{\omega} \widehat{\gamma}_{\mathfrak{u}}$, i.e., $\widehat{\gamma}_{\mathfrak{u}}=\Theta\left(\gamma_{\mathfrak{u}}\right)$.

The following result for finite-order weights was presented in 22 .

Theorem 3.1 ([22, Thm. 5(b)]). Let $\gamma$ be finite-order weights of order $\omega$. Let $\$(k)=\Omega\left(k^{s}\right)$ with $s>0$. The integration problem $\mathcal{I}_{\infty}$ is polynomially tractable if and only if decay $_{\boldsymbol{\gamma}, \omega}>1$. When this holds, then the tractability exponent $p^{*}$ satisfies

$$
\max \left\{1, \frac{2 \min \{1, s / \omega\}}{\text { decay }_{\boldsymbol{\gamma}, \omega}-1}\right\} \leq p^{*} \leq \max \left\{1, \frac{2}{\text { decay }_{\boldsymbol{\gamma}, \omega}-1}\right\}
$$


In particular, we have $p^{*}=1$ for decay $_{\boldsymbol{\gamma}, \omega} \geq 3$ and

$$
p^{*}=\frac{2}{\min \left\{3, \operatorname{decay}_{\boldsymbol{\gamma}, \omega}\right\}-1} \text { for } s \geq \omega .
$$

Thus the exact value of the exponent of tractability is known if decay ${ }_{\gamma, \omega} \geq 3$ or if $s \geq \omega$. It was left open in 22 what is the exact value of the exponent of tractability if the cost function satisfies $\$(k)=\Theta\left(k^{s}\right)$ for some $s \in[0, \omega)$ and if decay $_{\boldsymbol{\gamma}, \omega} \in(1,3)$. We believe that the case $s \in[0, \omega)$ is in fact the more important one. Clearly, the dependence of the cost function $\$(k)$ on the numbers of non-zero variables could be very different in different applications. But it seems reasonable to assume that in most applications $\$(k)$ depends linearly or slightly worse on $k$. This assumption means, in particular, that $s \in[0, \omega)$ for all $\omega>1$. (The case $\omega=1$ is not very interesting, since different variables do not interact. We provide a complete solution for this case in Remark 3.13.) Another reason for the importance of the case $s \in[0, \omega)$ are infinite-order weights. We may view infinite-order weights as the limiting case of finite-order weights, whose order $\omega$ tends to infinity. In the limiting process we finally have $s \in[0, \omega)$ for $\omega$ sufficiently large. We will use this observation in Section 4 , where we extend our results for finite-order weights to infinite-order weights.

In this section we improve (20). For general finite-order weights we improve the lower bound and, with the help of a new multilevel algorithm, for three newly defined classes of finite-order weights, also the upper bound. These lower and upper bounds match if decay $\boldsymbol{\gamma}, \boldsymbol{\omega}_{\text {is }}$ large or small enough. For finite-intersection weights (see Definition 3.5) we will provide the exact exponent of tractability for all values of $s$ and decay ${ }_{\boldsymbol{\gamma}, \omega}$.

3.1. Lower bounds for finite-order weights. Let us start by making an observation. The upper bound in Theorem 3.1 holds also for $s=0$. Indeed, as pointed out in [22], the special form of the cost function $\$$ does not play a major role in the upper bound for the exponent of tractability in (20). This is due to the fact that the proof of the upper bound in 22] relies on a linear algorithm that only uses function values at points with at most $\omega$ non-zero components. If we restrict ourselves to this class of linear algorithms, it can be shown that the upper bound in (201) is indeed sharp. More generally, we have the following result:

Theorem 3.2. Let $\$$ be an arbitrary cost function as defined in (9). Let $\gamma$ be finite-order weights of order $\omega$ that satisfy decay ${ }_{\boldsymbol{\gamma}, \omega}>1$. Let $B \geq \omega$ be a natural number. If we restrict ourselves to linear algorithms that use only function evaluations at points with at most $B$ non-zero components, i.e., to algorithms $\mathcal{Q}$ of the form (10) with $\max _{i \in[n]}\left|\mathfrak{v}_{i}\right| \leq B$, then the corresponding exponent $p_{B}^{*}$ of polynomial tractability is given by

$$
p_{B}^{*}=\max \left\{1, \frac{2}{\text { decay }_{\boldsymbol{\gamma}, \omega}-1}\right\} .
$$

Proof. We adapt the proof approach of [22, Thm. 5(b)]. Let $\mathcal{Q}$ be of the form (10) with $\max _{i \in[n]}\left|\mathfrak{v}_{i}\right| \leq B$. Its cost is given by (11). We obtain (see (13) and (12)),

$$
\mathcal{Q}(f)=\sum_{j=0}^{\infty} \mathcal{Q}_{\mathfrak{u}_{j}}\left(f_{\mathfrak{u}_{j}}\right)=\sum_{j=0}^{\infty} \sum_{\ell=1}^{n_{j}} a_{j_{\ell}} f_{\mathfrak{u}_{j}}\left(\boldsymbol{x}_{\mathfrak{u}_{j}}^{\left(j_{\ell}\right)} ; \mathbf{0}\right),
$$


where the sets $\mathfrak{u}_{j}=\mathfrak{u}_{j}(\omega)$ are defined as in Section 2.1] $n_{j}=\left|\left\{\mathfrak{v}_{i} \mid \mathfrak{u}_{j} \subseteq \mathfrak{v}_{i}\right\}\right|$, and $\left\{\mathfrak{v}_{i} \mid \mathfrak{u}_{j} \subseteq \mathfrak{v}_{i}\right\}=\left\{\mathfrak{v}_{j_{1}}, \ldots, \mathfrak{v}_{j_{n_{j}}}\right\}$.

The integration problem over $H\left(K_{\mathfrak{u}_{j}}\right), \mathfrak{u}_{j} \neq \emptyset$, is at least as hard as the univariate case. More precisely, there exist a $b>0$ and for each $j$ a function $f_{\mathfrak{u}_{j}}^{*} \in H\left(K_{\mathfrak{u}_{j}}\right)$ such that

$$
\begin{aligned}
\left|I_{\mathfrak{u}_{j}}\left(f_{\mathfrak{u}_{j}}^{*}\right)-\mathcal{Q}_{\mathfrak{u}_{j}}\left(f_{\mathfrak{u}_{j}}^{*}\right)\right| & \geq b\left(n_{j}+1\right)^{-1} 3^{-\left|\mathfrak{u}_{j}\right| / 2}\left\|f_{\mathfrak{u}_{j}}^{*}\right\|_{H\left(K_{\mathfrak{u}_{j}}\right)} \\
& =b\left(n_{j}+1\right)^{-1} \gamma_{\mathfrak{u}_{j}}^{1 / 2} 3^{-\left|\mathfrak{u}_{j}\right| / 2}\left\|f_{\mathfrak{u}_{j}}^{*}\right\|_{\mathcal{H}_{\gamma}}
\end{aligned}
$$

see (6). Thus we get from identity (14) the bound

$$
\left[e\left(\mathcal{Q} ; \mathcal{H}_{\gamma}\right)\right]^{2} \geq b^{2} \sum_{j=1}^{\infty} \frac{\widehat{\gamma}_{\mathfrak{u}_{j}}}{\left(n_{j}+1\right)^{2}}
$$

on the worst-case error of the algorithm $\mathcal{Q}$. Due to $\$(k) \geq 1$ for all $k \in \mathbb{N}_{0}$, we have $n \leq \operatorname{cost}(\mathcal{Q})$. Put $N:=\operatorname{cost}(\mathcal{Q})$. Then

$$
\begin{aligned}
\sum_{j=0}^{\infty} n_{j} & =\sum_{i=1}^{n}\left|\left\{j \in \mathbb{N}_{0} \mid \mathfrak{u}_{j} \subseteq \mathfrak{v}_{i}\right\}\right| \leq \sum_{i=1}^{n} \sum_{\ell=0}^{\min \left\{\left|\mathfrak{v}_{i}\right|, \omega\right\}}\left(\begin{array}{c}
\left|\mathfrak{v}_{i}\right| \\
\ell
\end{array}\right) \leq \sum_{i=1}^{n} \sum_{\ell=0}^{\min \left\{\left|\mathfrak{v}_{i}\right|, \omega\right\}} \frac{B^{\omega}}{\ell !} \\
& \leq \sum_{i=1}^{n} \mathrm{e} B^{\omega}=\mathrm{e} B^{\omega} n \leq \mathrm{e} B^{\omega} N
\end{aligned}
$$

Hence we have $\sum_{j=0}^{\infty} n_{j} \leq S$ with $S:=\left\lfloor\mathrm{e} B^{\omega} N\right\rfloor$.

Put $M:=\left|\left\{n_{j} \mid n_{j} \neq 0\right\}\right|$. We have $M \leq S$. To minimize our lower bound (21) for $\left[e\left(\mathcal{Q} ; \mathcal{H}_{\boldsymbol{\gamma}}\right)\right]^{2}$, it is clearly the best to choose those $n_{j} \geq 1$ whose corresponding weights $\widehat{\gamma}_{\mathfrak{u}_{j}}$ are largest, i.e., to choose $n_{1}, \ldots, n_{M} \geq 1$. Due to these observations we get

$$
e_{\gamma, B}(N) \geq b \min _{M=1,2, \ldots, S}\left(\sum_{j=1}^{M} \frac{\widehat{\gamma}_{\mathfrak{u}_{j}}}{\left(n_{j}+1\right)^{2}}+\operatorname{tail}_{\boldsymbol{\gamma}, \omega}(M)\right)^{1 / 2},
$$

where $e_{\gamma, B}(N)$ is the $N$ th minimal worst case error that can be achieved by algorithms $\mathcal{Q}$ of the form (10) with $\max _{i \in[n]}\left|\mathfrak{v}_{i}\right| \leq B$. Since decay $\boldsymbol{\gamma}_{\boldsymbol{\gamma}, \omega}>1$, we know from Theorem 3.1 that $\mathcal{I}_{\infty}$ is tractable. As already explained, the upper bound in (20) holds for general cost functions, and the algorithm used to establish it uses only function evaluations at points with at most $\omega$ non-zero components; see 22 , Thm. 5]. Hence $p_{B}^{*}<\infty$, and we have $e_{\boldsymbol{\gamma}, B}(N)=O\left(N^{-1 / p}\right)$ for any $p>p_{B}^{*}$. Due to the monotonicity of the $\widehat{\gamma}_{\mathfrak{u}_{j}}, j=1,2, \ldots$, and (23) we get

$$
S \widehat{\gamma}_{\mathfrak{u}_{2 S}} \leq \sum_{j=S+1}^{2 S} \widehat{\gamma}_{\mathfrak{u}_{j}} \leq \operatorname{tail}_{\boldsymbol{\gamma}, \omega}(S) \leq b^{-2}\left[e_{\boldsymbol{\gamma}, B}(N)\right]^{2}=O\left(N^{-2 / p}\right)
$$

We get $\widehat{\gamma}_{\mathfrak{u}_{2 S}}=O\left(S^{-1-2 / p}\right)$, which implies decay $\boldsymbol{\gamma}, \omega_{\boldsymbol{\omega}} \geq 1+2 / p_{B}^{*}$. In this case we thus have

$$
p_{B}^{*} \geq \frac{2}{\text { decay }_{\boldsymbol{\gamma}, \omega}-1} .
$$

We also have $p_{B}^{*} \geq 1$, since this even holds in the one-dimensional case. This completes the proof. 
With the help of the quantities $t_{\sigma}^{*}, \sigma \leq \omega$, from Definition 2.2 and the following remark we are able to improve the lower bound for general linear algorithms in (20).

Remark 3.3. If $\tilde{\gamma}$ is another set of weights satisfying $\tilde{\gamma}_{\mathfrak{u}} \leq \gamma_{\mathfrak{u}}$ for all finite $\mathfrak{u} \subset \mathbb{N}$, then the corresponding reproducing kernel Hilbert space $\mathcal{H}_{\tilde{\gamma}}$ has a larger norm than $\mathcal{H}_{\gamma}$, i.e.,

$$
\|f\|_{\mathcal{H}_{\tilde{\gamma}}} \geq\|f\|_{\mathcal{H}_{\gamma}} \text { for all } f \in \mathcal{H}_{\tilde{\gamma}}
$$

see (5). In particular, $\mathcal{H}_{\tilde{\gamma}} \subseteq \mathcal{H}_{\boldsymbol{\gamma}}$, and the unit ball of $\mathcal{H}_{\tilde{\gamma}}$ is contained in the unit ball of $\mathcal{H}_{\boldsymbol{\gamma}}$. Hence

$$
e\left(\mathcal{Q} ; \mathcal{H}_{\tilde{\gamma}}\right) \leq e\left(\mathcal{Q}, \mathcal{H}_{\boldsymbol{\gamma}}\right) \text { for any algorithm } \mathcal{Q} \text {. }
$$

This shows that the infinite-dimensional integration problem over $\mathcal{H}_{\tilde{\gamma}}$ is at most as hard as the one over $\mathcal{H}_{\boldsymbol{\gamma}}$, and consequently, the tractability exponent $p^{*}(\tilde{\gamma})$ for the weighted space $\mathcal{H}_{\tilde{\gamma}}$ will be at most as large as the exponent $p^{*}(\gamma)$ for the weighted space $\mathcal{H}_{\boldsymbol{\gamma}}$.

Theorem 3.4. Let $\$(k)=\Omega\left(k^{s}\right)$ for some $s \geq 0$. Let $\gamma$ be finite-order weights of order $\omega$ satisfying decay $_{\boldsymbol{\gamma}, \omega}>1$. Then the exponent of tractability satisfies

$$
p^{*} \geq \max \left\{1, \max _{\sigma \in[\omega]} \frac{2 \min \left\{1, s / t_{\sigma}^{*}\right\}}{\operatorname{decay}_{\boldsymbol{\gamma}, \sigma}-1}\right\} .
$$

Proof. The proof follows the lines of the proof of Theorem 3.2, We explain here only the proof steps that differ. For $s=0$ inequality (25) becomes $p^{*} \geq 1$, which we know to be true even in the one-dimensional case. So let $s>0$ and let $\mathcal{Q}$ be of the form (10) (but this time $\max _{i \in[n]}\left|\mathfrak{v}_{i}\right|$ can be arbitrarily large). Let $n_{j}=\left|\left\{\mathfrak{v}_{i} \mid \mathfrak{u}_{j} \subseteq \mathfrak{v}_{i}\right\}\right|$. There exists a $b>0$ such that

$$
\left[e\left(\mathcal{Q} ; \mathcal{H}_{\gamma}\right)\right]^{2} \geq b^{2} \sum_{j=1}^{\infty} \frac{\widehat{\gamma}_{\mathfrak{u}_{j}}}{\left(n_{j}+1\right)^{2}} .
$$

Due to our assumption $\$(k)=\Omega\left(k^{s}\right)$, there exists a constant $c>0$ such that

$$
\sum_{i=1}^{n}\left|\mathfrak{v}_{i}\right|^{s} \leq c \sum_{i=1}^{n} \$\left(\left|\mathfrak{v}_{i}\right|\right)=c \operatorname{cost}(\mathcal{Q}) .
$$

Put $N:=\operatorname{cost}(\mathcal{Q})$. With Jensen's inequality we get for $t>t_{\omega}^{*}$ and some $C_{t}>0$,

$$
\begin{aligned}
\sum_{j=1}^{\infty} n_{j} & =\sum_{i=1}^{n}\left|\left\{j \in \mathbb{N} \mid \mathfrak{u}_{j} \subseteq \mathfrak{v}_{i}\right\}\right| \leq \sum_{i=1}^{n} C_{t}\left|\mathfrak{v}_{i}\right|^{t} \\
& \leq C_{t}\left(\sum_{i=1}^{n}\left|\mathfrak{v}_{i}\right|^{s}\right)^{1 / \min \{1, s / t\}} \leq C_{t}(c N)^{1 / \min \{1, s / t\}} .
\end{aligned}
$$

This leads to $\sum_{j=1}^{\infty} n_{j} \leq S$ with $S:=\left\lfloor C_{t}(c N)^{1 / \min \{1, s / t\}}\right\rfloor$.

Put $M:=\left|\left\{n_{j} \mid n_{j} \neq 0\right\}\right|$. We have $M \leq S$, and get

$$
e_{\gamma}(N) \geq b \min _{M=1,2, \ldots, S}\left(\sum_{j=1}^{M} \frac{\widehat{\gamma}_{\mathfrak{u}_{j}}}{\left(n_{j}+1\right)^{2}}+\operatorname{tail}_{\boldsymbol{\gamma}, \omega}(M)\right)^{1 / 2} .
$$

Since decay $_{\boldsymbol{\gamma}, \omega}>1, p^{*}$ is finite. For any $p>p^{*}$ we have $e_{\boldsymbol{\gamma}}(N)=O\left(N^{-1 / p}\right)$. Thus we obtain $S \widehat{\gamma}_{\mathfrak{u}_{2 S}} \leq O\left(N^{-2 / p}\right)$. This leads to $\widehat{\gamma}_{\mathfrak{u}_{2 S}}=O\left(S^{-1-\frac{2}{p} \min \{1, s / t\}}\right)$, which 
implies decay $_{\boldsymbol{\gamma}, \omega} \geq 1+2 \min \left\{1, s / t_{\omega}^{*}\right\} / p^{*}$. Since we already know that $p^{*} \geq 1$, we get

$$
p^{*} \geq \max \left\{1, \frac{2 \min \left\{1, s / t_{\omega}^{*}\right\}}{\text { decay }_{\boldsymbol{\gamma}, \omega}-1}\right\} .
$$

Now let $\sigma \in[\omega]$, and let $p_{\sigma}^{*}=p_{\sigma}^{*}\left(\gamma^{(\sigma)}\right)$ be the exponent of tractability for the infinite-dimensional integration problem over the space $\mathcal{H}_{\gamma^{(\sigma)}}$. Since $\gamma_{\mathfrak{u}}^{(\sigma)} \leq \gamma_{\mathfrak{u}}$ for all finite $\mathfrak{u} \subset \mathbb{N}$ and decay $\boldsymbol{\gamma}, \sigma \geq$ decay $_{\boldsymbol{\gamma}, \omega}>1$, we have, due to Remark 3.3 and (27) (applied to the cut-off weights $\gamma^{(\sigma)}$ ),

$$
p^{*} \geq p_{\sigma}^{*} \geq \max \left\{1, \frac{2 \min \left\{1, s / t_{\sigma}^{*}\right\}}{\operatorname{decay}_{\boldsymbol{\gamma}, \sigma}-1}\right\} .
$$

It is straightforward to extend Theorem 3.4 to arbitrary weights; see Corollary 4.1 .

Notice that we have on the one hand $t_{1}^{*} \leq t_{2}^{*} \leq \cdots \leq t_{\omega}^{*}$, and on the other hand decay $_{\boldsymbol{\gamma}, 1} \geq$ decay $_{\boldsymbol{\gamma}, 2} \geq \cdots \geq$ decay $_{\boldsymbol{\gamma}, \boldsymbol{\omega}}$. Thus it is not a priori clear for which $\sigma \in[\omega]$ the maximum in (25) is taken. As we shall see, this actually varies for different classes of weights.

In the following three subsections, we will illustrate our lower bound for three classes of finite-order weights. For finite-intersection weights, we have $t_{\omega}^{*}=1$ and the maximum in (25) is taken for $\sigma=\omega$. In the case of finite-product weights, we have $t_{\omega}^{*}=\omega$ and the maximum is taken for $\sigma=1$. In both cases, (25) gives us a lower bound superior to the lower bound in (20). For lexicographically-ordered weights of order $\omega$, we have $t_{\omega}^{*}=\omega$ and the maximum in (25) is taken for $\sigma=\omega$. In this case, (25) does not improve on (20). Nevertheless, we shall see that this bound is sharp if decay $\boldsymbol{\gamma}, \boldsymbol{\omega}_{\boldsymbol{\omega}}$ is fast or slow enough.

\subsubsection{Lower bound for finite-intersection weights.}

Definition 3.5. Let $\rho \in \mathbb{N}$. The finite-order weights $\left\{\gamma_{\mathfrak{u}_{i}}\right\}_{i \in \mathbb{N}}$ are called finiteintersection weights with intersection degree at most $\rho$ if we have

$$
\left|\left\{j \in \mathbb{N} \mid \mathfrak{u}_{i} \cap \mathfrak{u}_{j} \neq \emptyset\right\}\right| \leq 1+\rho \text { for all } i \in \mathbb{N} .
$$

Note that for finite-order weights condition (28) is equivalent to the following condition: There exists an $\eta \in \mathbb{N}$ such that

$$
\left|\left\{i \in \mathbb{N} \mid k \in \mathfrak{u}_{i}\right\}\right| \leq \eta \quad \text { for all } k \in \mathbb{N} .
$$

Indeed, if (28) is satisfied, then (29) holds with $\eta \leq 1+\rho$, and if (29) is satisfied, then (28) holds with $\rho \leq(\eta-1) \omega$.

A subclass of the finite-intersection weights are the finite-diameter weights proposed by Creutzig; see, e.g., 31. The weights $\gamma=\left\{\gamma_{\mathfrak{u}}\right\}_{\mathfrak{u} \subset \mathbb{N} ;|\mathfrak{u}|<\infty}$ are called finitediameter weights if there exists an integer $q \geq 1$ such that

$$
\gamma_{\mathfrak{u}}=0 \text { for all finite } \mathfrak{u} \subset \mathbb{N} \text { with } \operatorname{diam}(\mathfrak{u}) \geq q,
$$

where $\operatorname{diam}(\mathfrak{u}):=\max _{k, \ell \in \mathfrak{u}}|k-\ell|$. By convention, $\operatorname{diam}(\emptyset)=0$. If finite-diameter weights satisfy (30) for some $q \in \mathbb{N}$, then they are obviously finite-order weights of order at most $q$ and finite-intersection weights of intersection degree upper-bounded, e.g., by $\sum_{\ell=1}^{q}\left(\begin{array}{c}3 q-2 \\ \ell\end{array}\right)$. Note that finite-intersection weights are not necessarily finitediameter weights. 
Now, let $\gamma$ be finite-intersection weights with intersection degree at most $\rho$. For a given finite set $\mathfrak{v} \subset \mathbb{N}$ we have

$$
\left|\left\{i \in \mathbb{N} \mid \mathfrak{u}_{i} \subseteq \mathfrak{v}\right\}\right| \leq(1+\rho)|\mathfrak{v}| .
$$

Hence $t_{\omega}^{*} \leq 1$. On the other hand, in the case where decay ${ }_{\boldsymbol{\gamma}, \omega}<\infty$, we have necessarily an infinite sequence $\left\{\mathfrak{u}_{j}\right\}_{j \in \mathbb{N}}$, implying $t_{\omega}^{*} \geq 1$. In this case $t_{\omega}^{*}=1$. In any case, (25) leads for finite-intersection weights to the lower bound

$$
p^{*} \geq \max \left\{1, \frac{2 \min \{1, s\}}{\operatorname{decay}_{\boldsymbol{\gamma}, \omega}-1}\right\},
$$

which improves on the lower bound in (20), and is for $\omega>\max \{1, s\}$ and decay ${ }_{\boldsymbol{\gamma}, \omega} \in$ $(1,1+2 \min \{1, s\})$ strictly better than (20). In Section 3.2 we will show that our lower bound for finite-intersection weights is actually sharp, i.e., the right-hand side of (31) turns out to be already the exact exponent of tractability.

3.1.2. Lower bound for lexicographically-ordered weights. To each set $\mathfrak{u} \subset \mathbb{N}$ with $|\mathfrak{u}|=\ell$ we assign the word $\varphi(\mathfrak{u}):=i_{1} i_{2} \ldots i_{\ell}$, where for $j \in[\ell]$ the number $i_{j}$ is the $j$ th-largest element of $\mathfrak{u}$. On the set of all finite words over the alphabet $\mathbb{N}$ we have the natural lexicographical order $\prec_{\text {lex }}$, where by convention the empty word should be the first (or "smallest") word.

Definition 3.6. We call weights $\gamma$ lexicographically-ordered weights of order $\omega$ if $\gamma_{\emptyset}=1, \gamma_{\mathfrak{u}}>0$ for all $\mathfrak{u} \subset \mathbb{N}$ with $|\mathfrak{u}| \leq \omega$, and

$$
\varphi\left(\mathfrak{u}_{i}\right) \prec_{\text {lex }} \varphi\left(\mathfrak{u}_{j}\right) \quad \text { for all } i, j \in \mathbb{N} \text { satisfying } i<j \text {. }
$$

This definition implies, e.g., that for all lexicographically-ordered weights $\gamma$ of order $\omega=3$ the ordered set system $\mathfrak{u}_{i}=\mathfrak{u}_{i}(\omega), i \in \mathbb{N}_{0}$, is given by $\mathfrak{u}_{0}=\emptyset$, $\mathfrak{u}_{1}=\{1\}, \mathfrak{u}_{2}=\{2\}, \mathfrak{u}_{3}=\{2,1\}, \mathfrak{u}_{4}=\{3\}, \mathfrak{u}_{5}=\{3,1\}, \mathfrak{u}_{6}=\{3,2\}, \mathfrak{u}_{7}=\{3,2,1\}$, $\mathfrak{u}_{8}=\{4\}, \mathfrak{u}_{9}=\{4,1\}, \mathfrak{u}_{10}=\{4,2\}, \mathfrak{u}_{11}=\{4,2,1\}, \mathfrak{u}_{12}=\{4,3\}, \mathfrak{u}_{13}=\{4,3,1\}$, $\mathfrak{u}_{14}=\{4,3,2\}, \mathfrak{u}_{15}=\{5\}, \ldots$

For lexicographically ordered weights $\gamma$ of order $\omega$ each finite $\mathfrak{v} \subset \mathbb{N}$ contains $\Theta\left(|\mathfrak{v}|^{\omega}\right)$ subsets, thus we have $t_{\omega}^{*}=\omega$. Furthermore, the corresponding cut-off weights $\gamma^{(\sigma)}$ are lexicographically-ordered weights of order $\sigma$ for each $\sigma \in[\omega]$. Due to (25), we get

$$
p^{*} \geq \max \left\{1, \frac{2 \min \{1, s / \omega\}}{\operatorname{decay}_{\gamma, \omega}-1}\right\}
$$

if $\$(k)=\Omega\left(k^{s}\right)$. This bound is the same as the lower bound in (20). We will prove in Section 3.2 an upper bound for lexicographically-ordered weights that improves on the upper bound in (20) and that demonstrates that the lower bound (32) is sharp if decay $\boldsymbol{\gamma}, \boldsymbol{\omega}_{\boldsymbol{\omega}}$ is large or small enough.

\subsubsection{Lower bound for finite-product weights.}

Definition 3.7. Let $\left\{\gamma_{j}\right\}_{j \in \mathbb{N}}$ be a sequence of non-negative real numbers satisfying $\gamma_{1} \geq \gamma_{2} \geq \ldots$. With the help of this sequence we define for $\omega \in \mathbb{N}$ finite-order weights $\gamma=\left\{\gamma_{\mathfrak{u}}\right\}_{\mathfrak{u} \subset \mathbb{N} ;|\mathfrak{u}|<\infty}$ of order (at most) $\omega$ by

$$
\gamma_{\mathfrak{u}}= \begin{cases}\prod_{j \in \mathfrak{u}} \gamma_{j} & \text { if }|\mathfrak{u}| \leq \omega \\ 0 & \text { otherwise }\end{cases}
$$


where we again use the convention that the empty product is 1 . Such weights we want to call finite-product weights of order (at most) $\omega$.

We may also be interested in the case of $\omega=\infty$, which corresponds to product weights, which were introduced by Sloan and Woźniakowski in [36. Weights $\gamma$ are called product weights if there exists a sequence of non-negative real numbers $\gamma_{1} \geq \gamma_{2} \geq \ldots$ such that $\gamma_{\mathfrak{u}}=\prod_{j \in \mathfrak{u}} \gamma_{j}$ for all finite $\mathfrak{u} \subset \mathbb{N}$.

The next lemma shows that the maximum over all $\sigma \in[\omega]$ that appears in our lower bound (25) is this time taken for $\sigma=1$.

Lemma 3.8. Let $\boldsymbol{\gamma}=\left\{\gamma_{\mathfrak{u}}\right\}_{\mathfrak{u} \subset \mathbb{N} ;|\mathfrak{u}|<\infty}$ be a set of finite-product weights of order $\omega$ or of bounded product weights (which, in both cases, do not necessarily have to satisfy condition (3) ). Then

$$
\text { decay }_{\boldsymbol{\gamma}, 1}=\text { decay }_{\boldsymbol{\gamma}, \sigma} \text { for all } \sigma \in \mathbb{N} \text {. }
$$

Proof. Let $\sigma \in \mathbb{N}$. Since decay $_{\boldsymbol{\gamma}, 1} \geq$ decay $_{\boldsymbol{\gamma}, \sigma} \geq 0$, it remains to show that decay $_{\boldsymbol{\gamma}, 1} \leq \operatorname{decay}_{\boldsymbol{\gamma}, \sigma}$. Since in the case decay $_{\boldsymbol{\gamma}, 1}=0$ we have nothing to show, let us assume that decay $_{\boldsymbol{\gamma}, 1}>0$. Let $p \in\left(0\right.$, decay $\left._{\boldsymbol{\gamma}, 1}\right)$. This implies $\sum_{j \in \mathbb{N}} \gamma_{j}^{1 / p}<\infty$. Thus we get

$$
\begin{aligned}
\sum_{j \in \mathbb{N}} \widehat{\gamma}_{\mathfrak{u}_{j}(\sigma)}^{1 / p} & \leq \prod_{j \in \mathbb{N}}\left(1+\left(\frac{\gamma_{j}}{3}\right)^{1 / p}\right)=\exp \left(\sum_{j \in \mathbb{N}} \ln \left(1+\left(\frac{\gamma_{j}}{3}\right)^{1 / p}\right)\right) \\
& \leq \exp \left(\sum_{j \in \mathbb{N}}\left(\frac{\gamma_{j}}{3}\right)^{1 / p}\right)<\infty,
\end{aligned}
$$

where we used the estimate $\ln (1+x) \leq x$, which holds for all non-negative $x$. This implies $\widehat{\gamma}_{\mathfrak{u}_{j}(\sigma)}=o\left(j^{-p}\right)$, since the sequence $\widehat{\gamma}_{\mathfrak{u}_{j}(\sigma)}, j \in \mathbb{N}$, is monotonic decreasing. Hence $p \leq$ decay $_{\boldsymbol{\gamma}, \sigma}$. Since we can choose $p$ arbitrarily close to decay ${ }_{\boldsymbol{\gamma}, 1}$ (which means, in the case decay $\boldsymbol{\gamma}, 1=\infty$, arbitrarily large), we obtain decay ${ }_{\boldsymbol{\gamma}, 1} \leq$ decay $_{\boldsymbol{\gamma}, \boldsymbol{\sigma}}$.

Recall that in the case where decay $_{\boldsymbol{\gamma}, \boldsymbol{\omega}}=\infty$ we already know from (20) that $p^{*}=1$. So let us assume that decay ${ }_{\boldsymbol{\gamma}, \omega}<\infty$, which, in particular, implies that all $\gamma_{j}$ are positive.

Then we obtain for all $\sigma \in[\omega]$ that $t_{\sigma}^{*}=\sigma$. Let $\$(k)=\Omega\left(k^{s}\right)$ for some $s \geq 0$. Due to Lemma 3.8 and Theorem 3.4 we have for finite-product weights with decay $\boldsymbol{\gamma}, \omega_{\boldsymbol{\omega}}>1$ that the exponent of tractability satisfies

$$
p^{*} \geq \max \left\{1, \frac{2 \min \{1, s\}}{\text { decay }_{\boldsymbol{\gamma}, 1}-1}\right\} .
$$

Note that (35) improves on the lower bound in (20) and is for $\omega>\max \{1, s\}$ and decay ${ }_{\gamma, \omega} \in(1,1+2 \min \{1, s\})$ strictly better than (201).

3.2. Upper bounds for finite-order weights. Now we will improve on the upper bound in (20) with the help of an algorithm that combines a multilevel idea with quasi-Monte Carlo integration. 
3.2.1. Description of the multilevel algorithm. Let us describe the general form of the algorithm we want to use more precisely:

Let $L_{0}:=0$, and let $L_{1}<L_{2}<L_{3}<\ldots$ be natural numbers, and let

$$
\mathfrak{v}_{k}^{(1)}:=\bigcup_{j \in\left[L_{k}\right]} \mathfrak{u}_{j} \text { and } \mathfrak{v}_{k}^{(2)}:=\left[L_{k}\right] \text { for } k \in \mathbb{N} \text {. }
$$

In the general case we will use the sets $\mathfrak{v}_{k}^{(1)}, k=1,2, \ldots$ In the special cases of lexicographically-ordered weights and finite- or infinite-product weights, it is more convenient to make use of the simple ordering of the corresponding set system $\mathfrak{u}_{j}$, $j \in \mathbb{N}$, (as defined in Section 2.1) and choose the sets $\mathfrak{v}_{k}^{(2)}$ for $k=1,2, \ldots$ In all definitions and results that hold for both choices of the $\mathfrak{v}_{k}^{(i)}, i=1,2$, we simply write $\mathfrak{v}_{k}$. We will choose the numbers $L_{1}, L_{2}, \ldots$ in general such that $\left|\mathfrak{v}_{k}\right|=\Theta\left(a^{k}\right)$ for some $a \in(1, \infty)$. (For the four different classes of weights considered in this paper, we will choose $L_{k}:=L\left\lceil a^{k-1}\right\rceil$, where $L \in \mathbb{N}$ is arbitrary. A "default choice" would be to take $L=1$ and $a=2$.) Let

$V_{1}:=\left\{j \in \mathbb{N} \mid \mathfrak{u}_{j} \subseteq \mathfrak{v}_{1}\right\} \quad$ and $\quad V_{k}:=\left\{j \in \mathbb{N} \mid \mathfrak{u}_{j} \subseteq \mathfrak{v}_{k}\right.$ and $\left.\mathfrak{u}_{j} \not \subset \mathfrak{v}_{k-1}\right\} \quad$ for $k \geq 2$.

Let us furthermore define for $m \in \mathbb{N}$,

$$
U(m):=\bigcup_{k=1}^{m} V_{k} \cup\{0\} .
$$

We put

$$
\mathcal{Q}_{k}(f):=\frac{1}{n_{k}} \sum_{j=1}^{n_{k}} f_{k}\left(\boldsymbol{t}_{\mathfrak{v}_{k}}^{(j, k)} ; \mathbf{0}\right)
$$

where

$$
f_{k}\left(\boldsymbol{t}_{\mathfrak{v}_{k}}^{(j, k)} ; \mathbf{0}\right):= \begin{cases}f\left(\boldsymbol{t}_{\mathfrak{v}_{1}}^{(j, 1)} ; \mathbf{0}\right) & \text { for } k=1, \\ f\left(\boldsymbol{t}_{\mathfrak{v}_{k}}^{(j, k)} ; \mathbf{0}\right)-f\left(\left(\boldsymbol{t}_{\mathfrak{v}_{k}}^{(j, k)}\right)_{\mathfrak{v}_{k-1}} ; \mathbf{0}\right) & \text { for } k \geq 2,\end{cases}
$$

and the numbers $n_{1} \geq n_{2} \geq \cdots \geq n_{m}$ and the points $\boldsymbol{t}_{\mathfrak{v}_{k}}^{(1, k)}, \ldots, \boldsymbol{t}_{\mathfrak{v}_{k}}^{\left(n_{k}, k\right)} \in[0,1]^{\left|\mathfrak{v}_{k}\right|}$ will be chosen later, depending on the weights $\gamma$. Define the algorithm $\mathcal{A}_{m}$ via

$$
\mathcal{A}_{m}(f):=\sum_{k=1}^{m} \mathcal{Q}_{k}(f)=\sum_{k=1}^{m} \frac{1}{n_{k}} \sum_{j=1}^{n_{k}} f_{k}\left(\boldsymbol{t}_{\mathfrak{v}_{k}}^{(k, j)} ; \mathbf{0}\right) .
$$

We assume in this section that $\$(k)=O\left(k^{s}\right)$ for some $s \geq 0$. Hence we have

$$
\operatorname{cost}\left(\mathcal{A}_{m}\right) \leq 2 \sum_{k=1}^{m} n_{k} \$\left(\left|\mathfrak{v}_{k}\right|\right)=O\left(\sum_{k=1}^{m} n_{k}\left|\mathfrak{v}_{k}\right|^{s}\right) .
$$

We obtain from (14) and (15)

$$
\left[e\left(\mathcal{A}_{m} ; \mathcal{H}_{\boldsymbol{\gamma}}\right)\right]^{2}=\sum_{j \in \mathbb{N}_{0}} \gamma_{\mathfrak{u}_{j}}\left[e\left(\mathcal{A}_{m, \mathfrak{u}_{j}} ; H\left(K_{\mathfrak{u}_{j}}\right)\right)\right]^{2},
$$

where $\mathcal{A}_{m, \mathfrak{u}_{j}}=\mathcal{A}_{m} \circ P_{\mathfrak{u}_{j}}=\sum_{k=1}^{m} \mathcal{Q}_{k, \mathfrak{u}_{j}}$. Note that

$$
e\left(\mathcal{A}_{m, \mathfrak{u}_{0}} ; H\left(K_{\mathfrak{u}_{0}}\right)\right)=e\left(\mathcal{A}_{m, \mathfrak{u}_{0}} ; \operatorname{span}\{1\}\right)=0,
$$

since $\mathcal{A}_{m}$ is exact on constant functions. Note furthermore that for all $j \in \mathbb{N}$ we have

$$
\mathcal{Q}_{k, \mathfrak{u}_{j}}(f)=0 \quad \text { whenever } j \notin V_{k} \text {. }
$$


Thus we get, using (15) and (16),

$$
\begin{aligned}
{\left[e\left(\mathcal{A}_{m} ; \mathcal{H}_{\boldsymbol{\gamma}}\right)\right]^{2} } & =\sum_{k=1}^{m} \sum_{j \in V_{k}} \gamma_{\mathfrak{u}_{j}}\left[e\left(Q_{k, \mathfrak{u}_{j}} ; H\left(K_{\mathfrak{u}_{j}}\right)\right)\right]^{2}+\sum_{\mathfrak{u}_{j} \notin U(m)} \gamma_{\mathfrak{u}_{j}}\left[e\left(\mathbf{0} ; H\left(K_{\mathfrak{u}_{j}}\right)\right)\right]^{2} \\
& =\sum_{k=1}^{m} \sum_{j \in V_{k}} \gamma_{\mathfrak{u}_{j}}\left[\operatorname{disc}_{2,\left|\mathfrak{u}_{j}\right|}^{*}\left(\left\{\overline{\boldsymbol{t}}_{\mathfrak{u}_{j}}^{(1, k)}, \ldots, \overline{\boldsymbol{t}}_{\mathfrak{u}_{j}}^{\left(n_{k}, k\right)}\right\}\right)\right]^{2}+\sum_{\mathfrak{u}_{j} \notin U(m)} \gamma_{\mathfrak{u}_{j}} 3^{-\left|\mathfrak{u}_{j}\right|} .
\end{aligned}
$$

To get a good error bound for our approximation of $\mathcal{I}_{\infty}$ by $\mathcal{A}_{m}$, we consequently need to find for each $k \in[m]$ point sets $\left\{\overline{\boldsymbol{t}}_{\mathfrak{v}_{k}}^{(1, k)}, \ldots, \overline{\boldsymbol{t}}_{\mathfrak{v}_{k}}^{\left(n_{k}, k\right)}\right\}$ whose projections onto the sets $\mathfrak{u}_{j}$ with $j \in V_{k}$ exhibit a small $L_{2}$-star discrepancy $\operatorname{disc}_{2,\left|\mathfrak{u}_{j}\right|}^{*}\left(\left\{\overline{\boldsymbol{t}}_{\mathfrak{u}_{j}}^{(1, k)}, \ldots\right.\right.$, $\left.\left.\overline{\boldsymbol{t}}_{\mathfrak{u}_{j}}^{\left(n_{k}, k\right)}\right\}\right)$. The problem of finding such point sets depends heavily on the weights we consider.

A reasonably good choice of point sets is provided by the next result. It follows from [35, Thm. 3(A)], which relies on constructive results for weighted Korobov spaces from [4]. We emphasize that Proposition 3.9 holds not only for finite-order, but in fact for general weights (which do not necessarily have to satisfy condition (3)). We will come back to this in Section 4 ,

Proposition 3.9. Let $\boldsymbol{\gamma}$ be arbitrary weights. Let $k \in \mathbb{N}$, and let $n_{k}$ be a prime number. Then there exists a point set $\left\{\boldsymbol{t}_{\mathfrak{v}_{k}}^{(1, k)}, \ldots, \boldsymbol{t}_{\mathfrak{v}_{k}}^{\left(n_{k}, k\right)}\right\} \subset[0,1]^{\left|\mathfrak{v}_{k}\right|}$ such that for all $\tau \in[1,2)$,

$$
\sum_{j \in V_{k}} \gamma_{\mathfrak{u}_{j}}\left[\operatorname{disc}_{2,\left|\mathfrak{u}_{j}\right|}^{*}\left(\left\{\overline{\boldsymbol{t}}_{\mathfrak{u}_{j}}^{(1, k)}, \ldots, \overline{\boldsymbol{t}}_{\mathfrak{u}_{j}}^{\left(n_{k}, k\right)}\right\}\right)\right]^{2} \leq F_{k, \tau, \boldsymbol{\gamma}}\left(n_{k}-1\right)^{-\tau},
$$

where, with $\zeta$ denoting the Riemann zeta function,

$$
F_{k, \tau, \gamma}:=\left(\sum_{j \in V_{k}} \gamma_{\mathfrak{u}_{j}}^{1 / \tau}\left(3^{-1 / \tau}+W_{\tau}\right)^{\left|\mathfrak{u}_{j}\right|}\right)^{\tau} \quad \text { and } \quad W_{\tau}:=\left(2 \pi^{2}\right)^{-1 / \tau}(2 \zeta(2 / \tau))
$$

Proof. Let us consider the space $\mathcal{H}_{\gamma^{*}}$, where the cut-off weights $\gamma^{*}$ are defined by $\gamma_{\mathfrak{u}}^{*}=\gamma_{\mathfrak{u}}$ if $\mathfrak{u}=\mathfrak{u}_{j}$ for some $j \in V_{k}$, and $\gamma_{\mathfrak{u}}^{*}=0$ otherwise. The space $\mathcal{H}_{\boldsymbol{\gamma}^{*}}$ can be identified with the weighted Sobolev space of $\left|\mathfrak{v}_{k}\right|$-variate functions anchored in zero and endowed with the weights $\left\{\gamma_{\mathfrak{u}}^{*}\right\}_{\mathfrak{u} \subseteq \mathfrak{v}_{k}}$; see, e.g., [35, Sect. 2]. Now [35, Thm. 3(A)] ensures the existence of a set $\left\{\boldsymbol{t}_{\mathfrak{v}_{k}}^{(\overline{1}, k)}, \ldots, \boldsymbol{t}_{\mathfrak{v}_{k}}^{\left(n_{k}, k\right)}\right\} \subset[0,1]^{\left|\mathfrak{v}_{k}\right|}$ such that $\left[e\left(\mathcal{Q}_{k} ; \mathcal{H}_{\boldsymbol{\gamma}^{*}}\right)\right]^{2} \leq \widetilde{F}_{k, \tau, \boldsymbol{\gamma}}\left(n_{k}-1\right)^{-\tau}$, where

$$
\widetilde{F}_{k, \tau, \boldsymbol{\gamma}}=\left(\sum_{\mathfrak{u} \subseteq \mathfrak{v}_{k}} W_{\tau}^{|\mathfrak{u}|}\left(\sum_{j \in V_{k} ; \mathfrak{u} \subseteq \mathfrak{u}_{j}} \gamma_{\mathfrak{u}_{j}} 3^{|\mathfrak{u}|-\left|\mathfrak{u}_{j}\right|}\right)^{1 / \tau}\right)^{\tau} .
$$

Due to (40) we have

$$
\sum_{j \in V_{k}} \gamma_{\mathfrak{u}_{j}}\left[\operatorname{disc}_{2,\left|\mathfrak{u}_{j}\right|}^{*}\left(\left\{\overline{\boldsymbol{t}}_{\mathfrak{u}_{j}}^{(1, k)}, \ldots, \overline{\boldsymbol{t}}_{\mathfrak{u}_{j}}^{\left(n_{k}, k\right)}\right\}\right)\right]^{2}=\left[e\left(\mathcal{Q}_{k} ; \mathcal{H}_{\boldsymbol{\gamma}^{*}}\right)\right]^{2} .
$$


Using Jensen's inequality, we obtain

$$
\begin{aligned}
\widetilde{F}_{k, \tau, \gamma} & \leq\left(\sum_{\mathfrak{u} \subseteq \mathfrak{v}_{k}} W_{\tau}^{|\mathfrak{u}|} \sum_{j \in V_{k} ; \mathfrak{u} \subseteq \mathfrak{u}_{j}} \gamma_{\mathfrak{u}_{j}}^{1 / \tau} 3^{\left(|\mathfrak{u}|-\left|\mathfrak{u}_{j}\right|\right) / \tau}\right)^{\tau} \\
& =\left(\sum_{j \in V_{k}} \gamma_{\mathfrak{u}_{j}}^{1 / \tau} 3^{-\left|\mathfrak{u}_{j}\right| / \tau} \sum_{\mathfrak{u} \subseteq \mathfrak{u}_{j}} W_{\tau}^{|\mathfrak{u}|} 3^{|\mathfrak{u}| / \tau}\right)^{\tau} .
\end{aligned}
$$

Now we have $\sum_{\mathfrak{u} \subseteq \mathfrak{u}_{j}} W_{\tau}^{|\mathfrak{u}|} 3^{|\mathfrak{u}| / \tau}=\left(1+3^{1 / \tau} W_{\tau}\right)^{\left|\mathfrak{u}_{j}\right|}$, which concludes the proof.

As demonstrated in [35, shifted rank-1 lattice rules, whose generators can be calculated efficiently by using the component-by-component (CBC) algorithm, satisfy (41). Unfortunately, these point sets are not fully constructive, since it is not known how to calculate a suitable shift $\Delta \in[0,1)^{\left|\mathfrak{v}_{k}\right|}$ efficiently.

We will use Proposition 3.9 for lexicographically-ordered weights, and for finite and infinite-product weights. For finite-intersection weights, however, we construct point sets explicitly, whose projections exhibit a discrepancy significantly smaller than the discrepancy guaranteed in Proposition 3.9. see Proposition 3.11.

3.2.2. Upper bounds for finite-intersection weights. Let $\gamma$ be finite-intersection weights of finite order $\omega$. In this case we can construct point sets or sequences of sample points that enable our algorithm $\mathcal{A}_{m}$ to provide a sharp upper bound for the exponent of tractability. Here, we take $\mathfrak{v}_{k}=\mathfrak{v}_{k}^{(1)}$ and $L_{k}:=L\left\lceil a^{k-1}\right\rceil$ for $k=1, \ldots, m$, where $L \in \mathbb{N}$ and $a \in(1, \infty)$ are fixed.

Our construction of optimal point sets is based on the following result.

Lemma 3.10. Let $\gamma$ be finite-intersection weights of finite order $\omega$. Let $\eta \in \mathbb{N}$ be such that the set system $\mathfrak{u}_{j}=\mathfrak{u}_{j}(\omega), j \in \mathbb{N}$, satisfies (29). Then there exists a mapping $\phi: \mathbb{N} \rightarrow[\eta(\omega-1)+1]$ such that for all $j \in \mathbb{N}$ the restriction $\left.\phi\right|_{\mathfrak{u}_{j}}$ is injective.

Proof. We will define $\phi$ inductively: Put $\phi(1)=1$. Let now $k \geq 2$ and let us assume that we have defined $\phi(\ell)$ for $\ell=1, \ldots, k-1$ such that $\left.\phi\right|_{\mathfrak{u}_{j} \cap[k-1]}$ is injective for all $j \in \mathbb{N}$ and takes only values in $[\eta(\omega-1)+1]$. For $k$ define $U_{k}:=\{\ell \in[k-1] \mid \exists j \in$ $\left.\mathbb{N}:\{\ell, k\} \subseteq \mathfrak{u}_{j}\right\}$. Since $k$ is contained in at most $\eta$ sets $\mathfrak{u}_{j}$, and since $\left|\mathfrak{u}_{j}\right| \leq \omega$ for all $j \in \mathbb{N}$, we see that $\left|U_{k}\right| \leq \eta(\omega-1)$. Thus $\phi(k):=\min \left([\eta(\omega-1)+1] \backslash \phi\left(U_{k}\right)\right)$ is a well-defined number in $[\eta(\omega-1)+1]$. Hence we have defined $\phi$ on $[k]$, and it is easy to see that $\left.\phi\right|_{\mathfrak{u}_{j} \cap[k]}$ is injective for every $j \in \mathbb{N}$ : If $k \notin \mathfrak{u}_{j}$, then $\left.\phi\right|_{\mathfrak{u}_{j} \cap[k]}=\left.\phi\right|_{\mathfrak{u}_{j} \cap[k-1]}$ is injective due to our induction hypothesis. If $k \in \mathfrak{u}_{j}$, then $\left.\phi\right|_{\mathfrak{u}_{j} \cap[k-1]}$ is injective due to our induction hypothesis. Furthermore, $\phi(k) \notin \phi\left(U_{k}\right)$, which in particular implies that $\phi(k) \notin \phi\left(\mathfrak{u}_{j} \cap[k-1]\right) \subseteq \phi\left(U_{k}\right)$.

Proposition 3.11. Let $\gamma$ be finite-intersection weights with finite order $\omega$. Let $\eta \in \mathbb{N}$ be such that the set system $\mathfrak{u}_{j}=\mathfrak{u}_{j}(\omega), j \in \mathbb{N}$, satisfies (29). Then there exists a sequence $Z_{\infty}=\left(\boldsymbol{z}_{\infty}^{(i)}\right)_{i \in \mathbb{N}}$ in $[0,1]^{\mathbb{N}}$ and a positive constant $C_{\eta, \omega}$ such that

$$
\operatorname{disc}_{2,\left|\mathfrak{u}_{j}\right|}^{*}\left(\left\{\boldsymbol{z}_{\infty, \mathfrak{u}_{j}}^{(1)}, \ldots, \boldsymbol{z}_{\infty, \mathfrak{u}_{j}}^{(n)}\right\}\right) \leq C_{\eta, \omega} n^{-1} \ln (n)^{\eta(\omega-1)+1} \quad \text { for all } j, n \in \mathbb{N} .
$$

Proof. In dimension $\eta(\omega-1)+1$ we find some suitable constant $C_{\eta, \omega}>0$ such that there exists a sequence $Z=\left(\boldsymbol{z}^{(i)}\right)_{i \in \mathbb{N}}$ in $[0,1]^{\eta(\omega-1)+1}$ satisfying

$$
\operatorname{disc}_{\infty, \eta(\omega-1)+1}^{*}\left(\left\{\boldsymbol{z}^{(1)}, \ldots, \boldsymbol{z}^{(n)}\right\}\right) \leq C_{\eta, \omega} n^{-1} \ln (n)^{\eta(\omega-1)+1} \text { for all } n \in \mathbb{N} .
$$


Indeed, efficiently computable low-discrepancy sequences often used in applications such as, e.g., the Faure, Halton, Niederreiter, Niederreiter-Xing or Sobol' sequence, achieve the convergence rate in (43); see, e.g., the papers [7, 14, 25, 27, 37] or the monograph 26.

From $Z$ we obtain an infinite-dimensional sequence $Z_{\infty}=\left(\boldsymbol{z}_{\infty}^{(i)}\right)_{i \in \mathbb{N}}$ by defining the $\nu$ th component of $\boldsymbol{z}_{\infty}^{(i)}$ via $z_{\infty, \nu}^{(i)}=z_{\phi(\nu)}^{(i)}$ for all $i, \nu \in \mathbb{N}$, where the mapping $\phi$ is as in Lemma 3.10, Let $j, n \in \mathbb{N}$. Since $\left.\phi\right|_{\mathfrak{u}_{j}}$ is injective, we have

$$
\begin{aligned}
& \operatorname{disc}_{2,\left|\mathfrak{u}_{j}\right|}^{*}\left(\left\{\boldsymbol{z}_{\infty, \mathfrak{u}_{j}}^{(1)}, \ldots, \boldsymbol{z}_{\infty, \mathfrak{u}_{j}}^{(n)}\right\}\right) \leq \operatorname{disc}_{\infty,\left|\mathfrak{u}_{j}\right|}^{*}\left(\left\{\boldsymbol{z}_{\infty, \mathfrak{u}_{j}}^{(1)}, \ldots, \boldsymbol{z}_{\infty, \mathfrak{u}_{j}}^{(n)}\right\}\right) \\
& \quad=\operatorname{disc}_{\infty,\left|\mathfrak{u}_{j}\right|}^{*}\left(\left\{\boldsymbol{z}_{\phi\left(\mathfrak{u}_{j}\right)}^{(1)}, \ldots, \boldsymbol{z}_{\phi\left(\mathfrak{u}_{j}\right)}^{(n)}\right\}\right) \leq \operatorname{disc}_{\infty, \eta(\omega-1)+1}^{*}\left(\left\{\boldsymbol{z}^{(1)}, \ldots, \boldsymbol{z}^{(n)}\right\}\right) \\
& \quad \leq C_{\eta, \omega} n^{-1} \ln (n)^{\eta(\omega-1)+1} .
\end{aligned}
$$

For any $n_{k}, k=1,2, \ldots$, we can construct points $\boldsymbol{t}_{\mathfrak{v}_{k}}^{(1, k)}, \ldots, \boldsymbol{t}_{\mathfrak{v}_{k}}^{\left(n_{k}, k\right)}$ explicitly such that

$$
\operatorname{disc}_{2,\left|\mathfrak{u}_{j}\right|}^{*}\left(\left\{\overline{\boldsymbol{t}}_{\mathfrak{u}_{j}}^{(1, k)}, \ldots, \overline{\boldsymbol{t}}_{\mathfrak{u}_{j}}^{\left(n_{k}, k\right)}\right\}\right) \leq C_{\eta, \omega} n_{k}^{-1} \ln \left(n_{k}\right)^{\eta(\omega-1)+1} \quad \text { for all } j \in V_{k}
$$

by simply choosing a sequence $Z_{\infty}$ as in Proposition 3.11 and $\overline{\boldsymbol{t}}_{\mathfrak{v}_{k}}^{(i, k)}:=\boldsymbol{z}_{\infty, \mathfrak{v}_{k}}^{(i)}$, $i=1, \ldots, n_{k}$. We can use the same sequence $Z_{\infty}$ for all values $m$ and $k$, regardless of the special value of $n_{k}, k=1, \ldots, m$. With this choice of sample points we get from (40) the error estimate

$$
\left[e\left(\mathcal{A}_{m} ; \mathcal{H}_{\boldsymbol{\gamma}}\right)\right]^{2} \leq C_{\eta, \omega, \delta} \sum_{k=1}^{m}\left(\sum_{j=L_{k-1}+1}^{L_{k}} \gamma_{\mathfrak{u}_{j}}\right) n_{k}^{2(\delta-1)}+\operatorname{tail}_{\boldsymbol{\gamma}, \omega}\left(L_{m}\right)
$$

for arbitrarily small $\delta \in(0,1)$ with a suitable constant $C_{\eta, \omega, \delta}$, depending only on $\delta$, $\eta$, and $\omega$. Notice that we implicitly used $n_{1} \geq n_{2} \geq \cdots \geq n_{m}$ to deduce (45), since it might be that for $L_{k-1}<j \leq L_{k}$ we have $\mathfrak{u}_{j} \subseteq \mathfrak{v}_{\ell}$ for some $\ell<k$.

Now let us try to find values of $n_{k}, k=1, \ldots, m$, which for given cost (essentially) minimize the right-hand side of (45). Note that here $\eta^{-1} L_{k} \leq\left|\mathfrak{v}_{k}\right| \leq \omega L_{k}$, i.e., $\left|\mathfrak{v}_{k}\right|=\Theta\left(L_{k}\right)$. Therefore (38) gives us

$$
\operatorname{cost}\left(\mathcal{A}_{m}\right)=O\left(\sum_{k=1}^{m} n_{k} L_{k}^{s}\right) .
$$

Put

$$
\sigma_{k}:=\sum_{j=L_{k-1}+1}^{L_{k}} \gamma_{\mathfrak{u}_{j}} \text { and } M:=\sum_{k=1}^{m} L_{k}^{s} .
$$

For a given $S>L^{s}$ let $m$ be such that $S \geq M$. We want to find the minimum $\boldsymbol{x}^{*}=\left(x_{1}^{*}, \ldots, x_{m}^{*}\right)$ of the function

$$
F(\boldsymbol{x})=\sum_{k=1}^{m} \sigma_{k} x_{k}^{2(\delta-1)} \quad \text { subject to the constraint } \sum_{k=1}^{m} x_{k} L_{k}^{s}=S .
$$

Due to Lagrange's multiplier theorem there exists a $\lambda \in \mathbb{R} \operatorname{such}$ that $\operatorname{grad} F\left(\boldsymbol{x}^{*}\right)=$ $\lambda\left(L_{1}^{s}, \ldots, L_{m}^{s}\right)$. This relation and the constraint imply that the minimum $\boldsymbol{x}^{*}$ is given by

$$
x_{k}^{*}=C \sigma_{k}^{\frac{1}{3-2 \delta}} L_{k}^{-\frac{s}{3-2 \delta}}, \quad \text { where } \quad C=S\left(\sum_{k=1}^{m} \sigma_{k}^{\frac{1}{3-2 \delta}} L_{k}^{\frac{2(1-\delta) s}{3-2 \delta}}\right)^{-1} .
$$


For $k=1,2, \ldots, m$ we choose $n_{k}:=\left\lceil x_{k}^{*}\right\rceil$. This leads to

$$
\operatorname{cost}\left(\mathcal{A}_{m}\right)=O\left(\sum_{k=1}^{m} n_{k} L_{k}^{s}\right)=O(S+M)=O(S) .
$$

Now we can estimate the different error terms that bound $\left[e\left(\mathcal{A}_{m} ; \mathcal{H}_{\gamma}\right)\right]^{2}$ in (45):

First we obtain

$$
\sum_{k=1}^{m} \sigma_{k} n_{k}^{2(\delta-1)} \leq S^{2(\delta-1)}\left(\sum_{k=1}^{m} \sigma_{k}^{\frac{1}{3-2 \delta}} L_{k}^{\frac{2(1-\delta) s}{3-2 \delta}}\right)^{3-2 \delta} .
$$

For $p \in\left(1\right.$, decay $\left._{\gamma, \omega}\right)$ we have $\gamma_{\mathfrak{u}_{j}}=O\left(j^{-p}\right)$, and consequently,

$$
\sigma_{k}=O\left(\sum_{j=L_{k-1}+1}^{L_{k}} j^{-p}\right)= \begin{cases}O\left(L_{k}^{1-p}\right) & \text { if } k \geq 2, \\ O(1) & \text { if } k=1 .\end{cases}
$$

This implies

$$
\sum_{k=1}^{m} \sigma_{k} n_{k}^{2(\delta-1)}=O\left(S^{2(\delta-1)}\left(1+L_{m}^{2(1-\delta) s+1-p}\right)\right) .
$$

Second, we obtain

$$
\operatorname{tail}_{\gamma, \omega}\left(L_{m}\right)=O\left(L_{m}^{1-p}\right)
$$

Thus we have altogether,

$$
\left[e\left(\mathcal{A}_{m} ; \mathcal{H}_{\gamma}\right)\right]^{2}=O\left(S^{2(\delta-1)}\left(1+L_{m}^{2(1-\delta) s+1-p}\right)+L_{m}^{1-p}\right) .
$$

Let $N:=\operatorname{cost}\left(\mathcal{A}_{m}\right)$. Note that $M=\Theta\left(L_{m}^{s}\right)$, and that $S \geq M$ implies $S=\Omega\left(L_{m}^{s}\right)$.

Case 1: decay $_{\boldsymbol{\gamma}, \omega}-1 \geq 2 s$. Then we have $2(1-\delta) s+1-p \leq 0$ for $p$ close enough to decay ${ }_{\gamma, \omega}$. Choosing $m$ in such a way that $S=\Theta\left(L_{m}^{\frac{p-1}{2(1-\delta)}}\right)$, we get

$$
e_{\boldsymbol{\gamma}}(N) \leq e\left(\mathcal{A}_{m} ; \mathcal{H}_{\gamma}\right)=O\left(L_{m}^{\frac{1-p}{2}}\right)=O\left(S^{-(1-\delta)}\right)=O\left(N^{-(1-\delta)}\right) .
$$

Since $\delta$ can be chosen arbitrarily small, the tractability exponent $p^{*}$ is at most 1 .

Case 2: decay ${ }_{\boldsymbol{\gamma}, \omega}-1<2 s$. Then we may choose $\delta$ small enough to obtain $2(1-\delta) s+1-p \geq 0$. Choosing $m$ in such a way that $S=\Theta\left(L_{m}^{s}\right)$, we get

$$
e_{\boldsymbol{\gamma}}(N) \leq e\left(\mathcal{A}_{m} ; \mathcal{H}_{\boldsymbol{\gamma}}\right)=O\left(L_{m}^{\frac{1-p}{2}}\right)=O\left(S^{-\frac{p-1}{2 s}}\right)=O\left(N^{-\frac{p-1}{2 s}}\right) .
$$

Since $p$ can be arbitrarily close to decay $_{\boldsymbol{\gamma}, \boldsymbol{\omega}}$, we get, in this case,

$$
p^{*} \leq \frac{2 s}{\operatorname{decay}_{\boldsymbol{\gamma}, \omega}-1} \text {. }
$$

If we have $s>t_{\omega}^{*}=1$ in case 2 , our specific choice of $L_{1}, L_{2}, \ldots$ does not allow our algorithm to match the lower bound. But in this case the algorithm from 22 , Thm. 5(a)], which uses only sample points with at most $\omega$ coordinates different from zero, proves

$$
p^{*} \leq \max \left\{1, \frac{2}{\text { decay }_{\boldsymbol{\gamma}, \omega}-1}\right\}
$$

see also Theorem 3.1 .

Altogether we proved the following theorem. 
Theorem 3.12. Let $\$(k)=O\left(k^{s}\right)$ for some $s \geq 0$. Let the finite-intersection weights $\boldsymbol{\gamma}$ of order $\omega$ satisfy decay ${ }_{\boldsymbol{\gamma}, \omega}>1$. Then the tractability exponent satisfies

$$
p^{*} \leq \max \left\{1, \frac{2 \min \{1, s\}}{\text { decay }_{\boldsymbol{\gamma}, \omega}-1}\right\} .
$$

In particular, if $\$(k)=\Theta\left(k^{s}\right)$, we have

$$
p^{*}=\max \left\{1, \frac{2 \min \{1, s\}}{\text { decay }_{\boldsymbol{\gamma}, \omega}-1}\right\} .
$$

Remark 3.13. Note that finite-order weights of order $\omega=1$ are (obviously) finiteintersection weights with intersection degree $\rho=0$. For them condition (29) holds for $\eta=1$. In this case we may use our multilevel algorithm together with a sequence $Z_{\infty}$ in $[0,1]^{\mathbb{N}}$, as described in Proposition 3.11. If we do not insist (on the practically very useful feature) that the quasi-Monte Carlo points we use for our multilevel algorithm stem from a sequence, we may simply use the point sets

$\left\{\boldsymbol{t}_{\mathfrak{v}_{k}}^{(1, k)}, \ldots, \boldsymbol{t}_{\mathfrak{v}_{k}}^{\left(n_{k}, k\right)}\right\} \subset[0,1]^{\left|\mathfrak{v}_{k}\right|}, \quad$ where $\boldsymbol{t}_{\mathfrak{v}_{k}, j}^{(\ell, k)}=\frac{2 \ell-1}{2 n_{k}}$ for $j \in V_{k}, \ell=1, \ldots, n_{k}$,

which leads to

$$
\operatorname{disc}_{2,\left|\mathfrak{u}_{j}\right|}^{*}\left(\left\{\overline{\boldsymbol{t}}_{\mathfrak{u}_{j}}^{(1, k)}, \ldots, \overline{\boldsymbol{t}}_{\mathfrak{u}_{j}}^{\left(n_{k}, k\right)}\right\}\right)=\frac{1}{\sqrt{12}} n_{k}^{-1} \quad \text { for all } j \in V_{k} \text { and all } k \in[m] .
$$

(Notice that $\left|\mathfrak{u}_{j}\right|=1$ for all $j \in \mathbb{N}$.) In the case $\$(k)=\Theta\left(k^{s}\right)$ we know the exact exponent of finite-order weights $\gamma$ of order $\omega=1$ and it is given by (49).

3.2.3. Upper bounds for lexicographically-ordered weights. Let $\gamma$ be lexicographicallyordered weights of finite order $\omega$. Let $L_{k}:=L\left\lceil a^{k-1}\right\rceil$ for $k \in \mathbb{N}$, where $L \in \mathbb{N}$ and $a \in(1, \infty)$ are fixed. Here we use our algorithm $\mathcal{A}_{m}$ with prime numbers $n_{1} \geq n_{2} \geq \cdots \geq n_{m}$ and the corresponding sample points $\boldsymbol{t}_{\mathfrak{v}_{k}}^{(1, k)}, \ldots, \boldsymbol{t}_{\mathfrak{v}_{k}}^{\left(n_{k}, k\right)}$, $k=1, \ldots, m$, from Proposition [3.9, where $\mathfrak{v}_{k}=\mathfrak{v}_{k}^{(2)}=\left[L_{k}\right]$. We define

$$
R_{0}:=0 \quad \text { and } \quad R_{k}:=\sum_{\sigma=1}^{\omega}\left(\begin{array}{c}
L_{k} \\
\sigma
\end{array}\right) \text { for } k=1, \ldots, m .
$$

We have $V_{k}=\left\{R_{k-1}+1, R_{k-1}+2, \ldots, R_{k}\right\}$ for all $k \geq 1$. Due to (40) and (41) we get for arbitrary $\tau \in\left[1, \min \left\{2, \operatorname{decay}_{\boldsymbol{\gamma}, \omega}\right\}\right)$ the error estimate

$$
\left[e\left(\mathcal{A}_{m} ; \mathcal{H}_{\boldsymbol{\gamma}}\right)\right]^{2} \leq \sum_{k=1}^{m} F_{k, \tau, \boldsymbol{\gamma}}\left(n_{k}-1\right)^{-\tau}+\operatorname{tail}_{\boldsymbol{\gamma}, \omega}\left(R_{m}\right)
$$

Furthermore,

$$
\begin{aligned}
F_{k, \tau, \gamma} & =\left(\sum_{j \in V_{k}} \gamma_{\mathfrak{u}_{j}}^{1 / \tau}\left(3^{-1 / \tau}+W_{\tau}\right)^{\left|\mathfrak{u}_{j}\right|}\right)^{\tau} \\
& \leq \max \left\{1,\left(3^{-1 / \tau}+W_{\tau}\right)^{\omega \tau}\right\}\left(\sum_{j \in V_{k}} \gamma_{\mathfrak{u}_{j}}^{1 / \tau}\right)^{\tau}=: \widehat{\sigma}_{k} .
\end{aligned}
$$

As done in the case of finite-intersection weights, let us try to find for given cost numbers $n_{k}, k=1, \ldots, m$, which essentially minimize our error estimate. Again, 
let $M:=\sum_{k=1}^{m} L_{k}^{s}$. For a given $S>2 L^{s}$ let $m$ be such that $S \geq 2 M$. Then the minimum $\boldsymbol{x}^{*}=\left(x_{1}^{*}, \ldots, x_{m}^{*}\right)$ of the function

$$
F(\boldsymbol{x})=\sum_{k=1}^{m} \widehat{\sigma}_{k}\left(x_{k}-1\right)^{-\tau} \quad \text { subject to the constraint } \sum_{k=1}^{m} x_{k} L_{k}^{s}=S
$$

is given by

$$
x_{k}^{*}=C \widehat{\sigma}_{k}^{\frac{1}{\tau+1}} L_{k}^{-\frac{s}{\tau+1}}+1, \quad \text { where } C=(S-M)\left(\sum_{k=1}^{m} \widehat{\sigma}_{k}^{\frac{1}{\tau+1}} L_{k}^{\frac{s \tau}{\tau+1}}\right)^{-1} .
$$

For $k=1,2, \ldots, m$ we choose $n_{k}$ to be the smallest prime greater than or equal to $x_{k}^{*}$. It is well known that for any $n \in \mathbb{N}$ there exists a prime number $p$ satisfying $n \leq p \leq 2 n$. Thus our choice of $n_{k}$ gives us $\left\lceil x_{k}^{*}\right\rceil \leq n_{k} \leq 2\left\lceil x_{k}^{*}\right\rceil$. This leads to

$$
N:=\operatorname{cost}\left(\mathcal{A}_{m}\right)=O\left(\sum_{k=1}^{m} n_{k} L_{k}^{s}\right)=O(2(S+M))=O(S) .
$$

Now let us estimate the different error terms in the bound of $\left[e\left(\mathcal{A}_{m} ; \mathcal{H}_{\boldsymbol{\gamma}}\right)\right]^{2}$ : First we obtain

$$
\sum_{k=1}^{m} \widehat{\sigma}_{k}\left(n_{k}-1\right)^{-\tau} \leq(S-M)^{-\tau}\left(\sum_{k=1}^{m} \widehat{\sigma}_{k}^{\frac{1}{\tau+1}} L_{k}^{\frac{s \tau}{\tau+1}}\right)^{\tau+1} .
$$

For $p \in\left(\tau\right.$, decay $\left.{ }_{\gamma, \omega}\right)$ we have $\gamma_{\mathfrak{u}_{j}}=O\left(j^{-p}\right)$. Since $R_{k}=\Theta\left(L_{k}^{\omega}\right)$ for all $k$, we get

$$
\widehat{\sigma}_{k}=O\left(\sum_{j=R_{k-1}+1}^{R_{k}} j^{-p / \tau}\right)^{\tau}= \begin{cases}O\left(L_{k}^{\omega(\tau-p)}\right) & \text { if } k \geq 2, \\ O(1) & \text { if } k=1\end{cases}
$$

This implies

$$
\sum_{k=1}^{m} \widehat{\sigma}_{k}\left(n_{k}-1\right)^{-\tau}=O\left(S^{-\tau}\left(1+L_{m}^{\omega(\tau-p)+s \tau}\right)\right) .
$$

Second, we obtain tail ${ }_{\gamma, \omega}\left(R_{m}\right)=O\left(L_{m}^{\omega(1-p)}\right)$. Thus we have altogether

$$
\left[e\left(\mathcal{A}_{m} ; \mathcal{H}_{\gamma}\right)\right]^{2}=O\left(S^{-\tau}\left(1+L_{m}^{\tau(\omega+s)-p \omega}\right)+L_{m}^{\omega(1-p)}\right) .
$$

Case 1: decay $\boldsymbol{\gamma}, \omega_{\boldsymbol{\omega}}>2(1+s / \omega)$. Choose $p$ large enough to ensure $p \geq 2(1+s / \omega)$. Then we get for all $\tau \in[1,2)$,

$$
\left[e\left(\mathcal{A}_{m} ; \mathcal{H}_{\gamma}\right)\right]^{2}=O\left(S^{-\tau}+L_{m}^{\omega(1-p)}\right) .
$$

Since $\omega(p-1) / \tau>s$, we can choose $m$ in such a way that $S=\Theta\left(L_{m}^{\omega(p-1) / \tau}\right)$, implying

$$
e_{\boldsymbol{\gamma}}(N) \leq e\left(\mathcal{A}_{m} ; \mathcal{H}_{\gamma}\right)=O\left(S^{-\tau / 2}\right)=O\left(N^{-\tau / 2}\right) .
$$

By taking $\tau$ arbitrarily close to 2 , we get the best possible exponent of tractability

$$
p^{*}=1 \text {. }
$$

Case 2: decay $_{\boldsymbol{\gamma}, \boldsymbol{\omega}} \leq 2(1+s / \omega)$. Hence $p \in(1,2(1+s / \omega))$. Let $r \geq s$, and let $m$ be such that $S=\Theta\left(L_{m}^{r}\right)$. Then

$$
\left[e\left(\mathcal{A}_{m} ; \mathcal{H}_{\gamma}\right)\right]^{2}=O\left(S^{-\mu}\right), \quad \text { where } \quad \mu:=\min \left\{\frac{\omega(p-1)}{r}, \tau, \tau+\frac{p \omega-\tau(\omega+s)}{r}\right\} .
$$


Choosing $r \geq \omega+s$ leads to $\mu \leq(p-1) /(1+s / \omega)$, and, for $p$ tending to decay ${ }_{\boldsymbol{\gamma}, \boldsymbol{\omega}}$, we cannot get an estimate for the exponent of tractability better than $p^{*} \leq$ $2(1+s / \omega) /\left(\operatorname{decay}_{\boldsymbol{\gamma}, \boldsymbol{\omega}}-1\right)$. As we will see, we can do better if we assume $r \in[s, s+\omega)$.

The value of $\tau$ that maximizes $\mu$ for $r \in[s, s+\omega)$ is $\tau=p \omega /(\omega+s)$. Here we have $p \omega /(\omega+s)<2$, so for $s>0$ the optimal choice of $\tau$ satisfying the constraint $\tau \in[1, \min \{2, p\})$ is given by

$$
\tau=\max \left\{1, \frac{p \omega}{\omega+s}\right\}
$$

and for $s=0$ we may choose $\tau$ arbitrarily close to $p$.

Subcase a: decay ${ }_{\boldsymbol{\gamma}, \boldsymbol{\omega}} \leq 1+s / \omega$. Then we have $p<1+s / \omega$ and consequently $\tau=1$. Hence

$$
\mu=\min \left\{\frac{\omega(p-1)}{r}, 1+\frac{p \omega-(\omega+s)}{r}\right\} .
$$

The choice $r=s$ maximizes $\mu$ and leads to $S=\Theta\left(L_{m}^{s}\right)$ and

$$
e_{\boldsymbol{\gamma}}(N) \leq e\left(\mathcal{A}_{m} ; \mathcal{H}_{\boldsymbol{\gamma}}\right)=O\left(S^{-\frac{\omega(p-1)}{2 s}}\right)=O\left(N^{-\frac{\omega(p-1)}{2 s}}\right) .
$$

Choosing $p$ arbitrarily close to decay ${ }_{\boldsymbol{\gamma}, \boldsymbol{\omega}}$ leads to

$$
p^{*} \leq \frac{2 s / \omega}{\operatorname{decay}_{\boldsymbol{\gamma}, \omega}-1} .
$$

Subcase b: decay ${ }_{\boldsymbol{\gamma}, \boldsymbol{\omega}}>1+s / \omega$. Then we may choose $p \geq 1+s / \omega$ and $\tau=$ $p \omega /(\omega+s)$. (Only in the case $s=0$ we have to choose $\tau=p-\varepsilon, \varepsilon>0$ arbitrarily small. But we do not discuss this case in detail, since it is obvious how the following argument has to be modified.) Hence

$$
\mu=\min \left\{\frac{\omega(p-1)}{r}, \frac{p \omega}{\omega+s}\right\} .
$$

The choice $r \in[s,(\omega+s)(p-1) / p]$ leads to $\mu=p \omega /(\omega+s)$ and

$$
e_{\boldsymbol{\gamma}}(N) \leq e\left(\mathcal{A}_{m} ; \mathcal{H}_{\gamma}\right)=O\left(S^{-\frac{p \omega}{2(\omega+s)}}\right)=O\left(N^{-\frac{p \omega}{2(\omega+s)}}\right) \text {. }
$$

Choosing $p$ arbitrarily close to decay $_{\boldsymbol{\gamma}, \omega}$ leads to

$$
p^{*} \leq \frac{2(1+s / \omega)}{\operatorname{decay}_{\boldsymbol{\gamma}, \omega}} .
$$

Since the algorithm used in [22, Thm. 5(a)] proves

$$
p^{*} \leq \max \left\{1, \frac{2}{\text { decay }_{\boldsymbol{\gamma}, \omega}-1}\right\},
$$

we established altogether the following theorem.

Theorem 3.14. Let $\$(k)=O\left(k^{s}\right)$ for some $s \geq 0$. Let the lexicographically-ordered weights $\gamma$ of finite order $\omega$ satisfy decay $_{\boldsymbol{\gamma}, \omega}>1$. Then the tractability exponent satisfies

$$
p^{*} \leq \max \left\{1,2 \min \left\{\max \left\{\frac{s / \omega}{\operatorname{decay}_{\boldsymbol{\gamma}, \omega}-1}, \frac{1+s / \omega}{\text { decay }_{\boldsymbol{\gamma}, \omega}}\right\}, \frac{1}{\operatorname{decay}_{\boldsymbol{\gamma}, \boldsymbol{\omega}}-1}\right\}\right\} .
$$

In particular, we have for $1<\operatorname{decay}_{\boldsymbol{\gamma}, \boldsymbol{\omega}} \leq 1+s / \omega$,

$$
p^{*} \leq \max \left\{1, \frac{2 \min \{1, s / \omega\}}{\text { decay }_{\boldsymbol{\gamma}, \boldsymbol{\omega}}-1}\right\},
$$


for $1+s / \omega<$ decay $_{\boldsymbol{\gamma}, \omega}<2(1+s / \omega)$,

$$
p^{*} \leq \max \left\{1,2 \min \left\{\frac{1}{\text { decay }_{\boldsymbol{\gamma}, \omega}-1}, \frac{1+s / \omega}{\text { decay }_{\boldsymbol{\gamma}, \omega}}\right\}\right\},
$$

and for $2(1+s / \omega) \leq$ decay $_{\boldsymbol{\gamma}, \omega}$,

$$
p^{*}=1 \text {. }
$$

If $\$(k)=\Theta\left(k^{s}\right)$ the lower bound (32) always matches the upper bound (55), except when $s<\omega$ and $1+s / \omega<\operatorname{decay}_{\boldsymbol{\gamma}, \boldsymbol{\omega}}<\min \{3,2(1+s / \omega)\}$.

3.2.4. Upper bounds for finite-product weights. Let $\gamma$ be finite-product weights of finite order $\omega$. Let $L_{k}:=L\left\lceil a^{k-1}\right\rceil$ for $k \in \mathbb{N}$, where $L \in \mathbb{N}$ and $a \in(1, \infty)$ are fixed. As for lexicographically-ordered weights, we use our algorithm $\mathcal{A}_{m}$ with the sample points $\boldsymbol{t}_{\mathfrak{v}_{k}}^{(1, k)}, \ldots, \boldsymbol{t}_{\mathfrak{v}_{k}}^{\left(n_{k}, k\right)}$ from Proposition 3.9, where $\mathfrak{v}_{k}=\mathfrak{v}_{k}^{(2)}=\left[L_{k}\right]$ for $k=1, \ldots, m$, and get for arbitrary $\tau \in\left[1, \min \left\{2\right.\right.$, decay $\left.\left._{\boldsymbol{\gamma}, 1}\right\}\right)$ the error estimate (50).

In the next lemma we present an estimate for $F_{k, \tau, \gamma}$, which does not only hold for finite-product weights, but also for (infinite-)product weights.

Lemma 3.15. Let $\boldsymbol{\gamma}$ be finite-product or product weights with $\operatorname{decay}_{\boldsymbol{\gamma}, 1}>1$. Then

$$
F_{k, \tau, \gamma}=O\left(\tilde{\sigma}_{k}\right), \text { where } \tilde{\sigma}_{k}:=\left(\sum_{j=L_{k-1}+1}^{L_{k}} \gamma_{j}^{1 / \tau}\right)^{\tau} .
$$

Proof. Put $W_{\tau}^{*}:=3^{-1 / \tau}+W_{\tau}$. Then

$$
\begin{aligned}
F_{k, \tau, \gamma} & \leq\left[\prod_{j=1}^{L_{k}}\left(1+\gamma_{j}^{1 / \tau} W_{\tau}^{*}\right)-\prod_{j=1}^{L_{k-1}}\left(1+\gamma_{j}^{1 / \tau} W_{\tau}^{*}\right)\right]^{\tau} \\
& =\left[\prod_{j=1}^{L_{k-1}}\left(1+\gamma_{j}^{1 / \tau} W_{\tau}^{*}\right)\left(\prod_{j=L_{k-1}+1}^{L_{k}}\left(1+\gamma_{j}^{1 / \tau} W_{\tau}^{*}\right)-1\right)\right]^{\tau} .
\end{aligned}
$$

Now

$$
\begin{aligned}
\prod_{j=L_{k-1}+1}^{L_{k}}\left(1+\gamma_{j}^{1 / \tau} W_{\tau}^{*}\right) & =\exp \left(\sum_{j=L_{k-1}+1}^{L_{k}} \ln \left(1+\gamma_{j}^{1 / \tau} W_{\tau}^{*}\right)\right) \\
& \leq \exp \left(W_{\tau}^{*} \sum_{j=L_{k-1}+1}^{L_{k}} \gamma_{j}^{1 / \tau}\right)
\end{aligned}
$$

implying

$$
\prod_{j=L_{k-1}+1}^{L_{k}}\left(1+\gamma_{j}^{1 / \tau} W_{\tau}^{*}\right)-1 \leq W_{\tau}^{*}\left(\sum_{j=L_{k-1}+1}^{L_{k}} \gamma_{j}^{1 / \tau}\right)(1+o(1)) \quad \text { as } k \rightarrow \infty .
$$

Since $\tau<$ decay $_{\boldsymbol{\gamma}, 1}$, we have

$$
\prod_{j=1}^{L_{k-1}}\left(1+\gamma_{j}^{1 / \tau} W_{\tau}^{*}\right) \leq \prod_{j=1}^{\infty}\left(1+\gamma_{j}^{1 / \tau} W_{\tau}^{*}\right)<\infty .
$$


Now the following theorem can easily be verified by following the proof of Theorem 3.14 where one just has to substitute $\omega$ by $1, R_{k}$ by $L_{k}$, and $\hat{\sigma}_{k}$ by $\tilde{\sigma}_{k}$ for $k=1, \ldots, m$.

Theorem 3.16. Let $\$(k)=O\left(k^{s}\right)$ for some $s \geq 0$. Let the finite-product weights $\gamma$ of finite order $\omega$ satisfy decay ${ }_{\boldsymbol{\gamma}, 1}>1$. Then the tractability exponent satisfies

$$
p^{*} \leq \max \left\{1,2 \min \left\{\max \left\{\frac{s}{\text { decay }_{\boldsymbol{\gamma}, 1}-1}, \frac{1+s}{\text { decay }_{\boldsymbol{\gamma}, 1}}\right\}, \frac{1}{\text { decay }_{\boldsymbol{\gamma}, 1}-1}\right\}\right\} .
$$

In particular, we have for $1<$ decay $_{\boldsymbol{\gamma}, 1} \leq 1+s$,

$$
p^{*} \leq \max \left\{1, \frac{2 \min \{1, s\}}{\operatorname{decay}_{\boldsymbol{\gamma}, 1}-1}\right\}
$$

for $1+s<$ decay $_{\boldsymbol{\gamma}, 1}<2(1+s)$,

$$
p^{*} \leq \max \left\{1,2 \min \left\{\frac{1}{\text { decay }_{\boldsymbol{\gamma}, 1}-1}, \frac{1+s}{\text { decay }_{\boldsymbol{\gamma}, 1}}\right\}\right\},
$$

and for $2(1+s) \leq$ decay $_{\boldsymbol{\gamma}, 1}$,

$$
p^{*}=1 \text {. }
$$

If $\$(k)=\Theta\left(k^{s}\right)$ the lower bound (35) always matches the upper bound (56), except when $s<1$ and $1+s<\operatorname{decay}_{\boldsymbol{\gamma}, 1}<\min \{3,2(1+s)\}$.

\section{From Finite-ORDER WEIGHTS TO ARBITRARY WEIGHTS}

In this section we apply the results and methods we provided in the case of finiteorder weights to obtain results for arbitrary weights, in particular, for infinite-order weights. As in the previous sections, we always assume that the weights $\gamma$ satisfy (3) and that there exists at least one finite, non-empty set $\mathfrak{u} \subset \mathbb{N}$ with $\gamma_{\mathfrak{u}}>0$.

4.1. Lower bounds for arbitrary weights. Let $\gamma$ be an arbitrary set of weights, and let $\sigma \in \mathbb{N}$. As explained in Remark 3.3, the infinite-dimensional integration problem is not harder for the cut-off weights $\gamma^{(\sigma)}$ than for the original weights $\gamma$. Thus any lower bound on the exponent of tractability $p_{\sigma}^{*}=p^{*}\left(\gamma^{(\sigma)}\right)$ corresponding to the cut-off weights $\gamma^{(\sigma)}$ holds also for the exponent of tractability $p^{*}=p^{*}(\gamma)$ for the original weights $\gamma$. Since cut-off weights $\gamma^{(\sigma)}$ are in particular finite-order weights of order at most $\sigma$, we get from Theorem 3.4 immediately the following result.

Corollary 4.1. Let $\gamma$ be arbitrary weights, and let $\$(k)=\Omega\left(k^{s}\right)$ for some $s \geq 0$. Let the integration problem $\mathcal{I}_{\infty}$ be tractable.

$A$ lower bound for the exponent of tractability $p^{*}$ is then given by

$$
p^{*} \geq \max \left\{1, \sup _{\sigma \in \mathbb{N}} \frac{2 \min \left\{1, s / t_{\sigma}^{*}\right\}}{\operatorname{decay}_{\boldsymbol{\gamma}, \sigma}-1}\right\} .
$$


4.1.1. Lower bound for product weights. Product weights are probably the most extensively studied type of weights in the literature of multivariate problems. If $\gamma$ are product weights, then the cut-off weights $\gamma^{(\sigma)}, \sigma \in \mathbb{N}$, are obviously finiteproduct weights of order at most $\sigma$.

Let us assume decay ${ }_{\gamma, 1}<\infty$, which in particular means that all $\gamma_{j}$ are positive. This implies $t_{\sigma}^{*}=\sigma$ for all $\sigma \in \mathbb{N}$. Now let $\$(k)=\Omega\left(k^{s}\right)$ for some $s \geq 0$. Then we get from Lemma 3.8 and Corollary 4.1 the following lower bound for the exponent of tractability $p^{*}$ for product weights $\gamma$ :

$$
p^{*} \geq \max \left\{1, \frac{2 \min \{1, s\}}{\operatorname{decay}_{\boldsymbol{\gamma}, 1}-1}\right\} .
$$

This lower bound for product weights was provided in [22, Sect. 3.3] for the case where $\gamma_{j}=j^{-\beta}$ for arbitrary $\beta>1$. The point we want to make here, is that this non-trivial result already follows from our results in the easier setting of finite-order weights. Note that in contrast the lower bound (58) for (infinite-)product weights does not imply our bound (35) for finite-product weights.

4.2. Upper bounds for arbitrary weights. Our algorithm $\mathcal{A}_{m}$ can in general be used for arbitrary weights to provide upper bounds for the exponent of tractability. For arbitrary weights $\gamma$ we may use the sets $\mathfrak{v}_{k}=\mathfrak{v}_{k}^{(1)}=\bigcup_{j \in\left[L_{k}\right]} \mathfrak{u}_{j}$ in (36). If $s$ is not too large, the numbers $L_{1}, L_{2}, L_{3}, \ldots$ should be chosen in such a way that $\left|\mathfrak{v}_{k}\right|$ grows exponentially, i.e., $\left|\mathfrak{v}_{k}\right|=\Theta\left(a^{k}\right)$ for some $a \in(1, \infty)$. For $k=1,2, \ldots, m$ the points $\left\{\boldsymbol{t}_{\mathfrak{v}_{k}}^{(1, k)}, \ldots, \boldsymbol{t}_{\mathfrak{v}_{k}}^{\left(n_{k}, k\right)}\right\}$ can always be chosen as in Proposition 3.9. which holds not only for finite-order weights, but also for arbitrary weights.

Here we confine ourselves to provide an explicit error bound in the case of product weights.

4.2.1. Upper bound for product weights. In this section we can use almost directly our results from the case of finite-product weights. The observation we have to make is the following:

In the whole derivation of Theorem 3.16 the only occasion where we benefited from the fact that we were working with finite-product weights was that we used the bound

$$
p^{*} \leq \max \left\{1, \frac{2}{\text { decay }_{\boldsymbol{\gamma}, 1}-1}\right\},
$$

from [22, Thm. 5(a)] (see also Theorem 3.1). The algorithm used there to establish (59) relies crucially on the fact that the weights under consideration are finiteorder weights. Thus we cannot expect (59) to hold. But our algorithm with the numbers $L_{k}=L\left\lceil a^{k-1}\right\rceil$ for some $L \in \mathbb{N}$ and $a \in(1, \infty)$, the sets of coordinates $\mathfrak{v}_{k}=\mathfrak{v}_{k}^{(2)}=\left[L_{k}\right]$, and the point sets $\left\{\boldsymbol{t}_{\mathfrak{v}_{k}}^{(1, k)}, \ldots, \boldsymbol{t}_{\mathfrak{v}_{k}}^{\left(n_{k}, k\right)}\right\}$ from Proposition 3.9 leads also in the case of (infinite-)product weights to the estimates (51), (52), and (53) with $\omega=1$.

Altogether, we get the following result.

Theorem 4.2. Let $\$(k)=O\left(k^{s}\right)$ for some $s \geq 0$. Let the product weights $\gamma$ satisfy decay $_{\boldsymbol{\gamma}, 1}>1$. Then the tractability exponent satisfies

$$
p^{*} \leq \max \left\{1, \frac{2 s}{\text { decay }_{\boldsymbol{\gamma}, 1}-1}, \frac{2(1+s)}{\text { decay }_{\boldsymbol{\gamma}, 1}}\right\} .
$$


In particular, we have for $1<$ decay $_{\boldsymbol{\gamma}, \boldsymbol{1}} \leq 1+s$,

$$
p^{*} \leq \frac{2 s}{\operatorname{decay}_{\boldsymbol{\gamma}, 1}-1},
$$

for $1+s<$ decay $_{\boldsymbol{\gamma}, 1}<2(1+s)$,

$$
p^{*} \leq \frac{2(1+s)}{\text { decay }_{\boldsymbol{\gamma}, 1}}
$$

and for $2(1+s) \leq$ decay $_{\boldsymbol{\gamma}, 1}$,

$$
p^{*}=1 \text {. }
$$

If $\$(k)=\Theta\left(k^{s}\right)$ the lower bound (58) matches the upper bound (60) in the case where decay ${ }_{\boldsymbol{\gamma}, 1} \geq 2(1+s)$, and in the case where $s \leq 1$ and $1+s \geq$ decay $_{\gamma, 1}$.

Remark 4.3. Let us compare the result of our algorithm to the results of the algorithms used in 22. The first algorithm studied there in Section 3.1 is a direct quasi-Monte Carlo approach called fixed dimension algorithm. Our algorithm reduces to the fixed dimension algorithm if for a required error guarantee $\varepsilon$ we choose $m=1$ and put $L_{1}=d(\varepsilon)$, where $d(\varepsilon)$ tends to infinity as $\varepsilon$ approaches zero; see [22, Sect. 3.1] for details.

If decay $_{\boldsymbol{\gamma}, \boldsymbol{1}}>1$, the fixed dimension algorithm leads, for product weights, to the following bound on the exponent of tractability:

$$
p^{*} \leq \max \left\{1, \frac{2}{\text { decay }_{\boldsymbol{\gamma}, 1}}\right\}+\frac{2 s}{\text { decay }_{\boldsymbol{\gamma}, 1}-1} .
$$

If $s=0$ or if decay ${ }_{\boldsymbol{\gamma}, 1}=\infty$ this is the same bound as provided by our algorithm. If $s>0$ and decay ${ }_{\boldsymbol{\gamma}, 1}<\infty$, then (60) is strictly smaller than (61).

The second algorithm studied in [22, Sect. 3.2] is called changing dimension algorithm.

If decay $\boldsymbol{\gamma}, 1_{1}>1$, the changing dimension algorithm leads, for product weights, to the following bound on the exponent of tractability:

$$
p^{*} \leq 1+\frac{2}{\text { decay }_{\boldsymbol{\gamma}, 1}-1} .
$$

In the case where decay ${ }_{\boldsymbol{\gamma}, 1} \geq 2(1+s)$ our algorithm leads to the best possible exponent 1 , while the changing dimension algorithm does only in the case decay ${ }_{\gamma, 1}=\infty$.

In the case $2(1+s)>$ decay $_{\boldsymbol{\gamma}, \boldsymbol{1}}>1+s$ our algorithm leads to a better result than the changing dimension algorithm if $2 \geq s$. If $2<s$, our algorithm is better as long as decay $\boldsymbol{\gamma}, 1_{1}>\delta(s)$, where

$$
\delta(s):=\frac{1}{2}+s+\sqrt{\frac{1}{4}+(s-2)(1+s)} .
$$

If $1+s<$ decay $_{\boldsymbol{\gamma}, 1}<\delta(s)$, then the changing dimension algorithm is better (which can, as already mentioned, only happen if $s>2$ ).

In the case $1+s \geq$ decay $_{\boldsymbol{\gamma}, 1}>1$, our algorithm leads to a better result than the changing dimension algorithm for decay $_{\boldsymbol{\gamma}, 1}>2 s-1$, while the changing algorithm leads to a better result if decay ${ }_{\boldsymbol{\gamma}, 1}<2 s-1$, which can only occur if $s>1$.

This means, in particular, that for $s \leq 1$ our algorithm leads in any case to a better or equal result than the changing dimension algorithm. 
Remark 4.4. More restrictive models of varying costs of function evaluations, called fixed and variable subspace sampling, are studied in [3, 18, 24, 28, 29. A discussion of both cost models can be found, e.g., in [24, Sect. 2]. We restate the variable subspace sampling model, which is similar to the (more generous) cost model from [22]. For subspaces

$$
\mathcal{X}_{1: d}:=\left\{x \in[0,1]^{\mathbb{N}} \mid x_{d+1}=x_{d+2}=\cdots=0\right\}
$$

of $[0,1]^{\mathbb{N}}$, the cost of an algorithm $\mathcal{Q}$ of the form (10) in the variable subspace model is

$$
\operatorname{cost}^{\operatorname{var}}(\mathcal{Q}):=\sum_{i=1}^{n}\left[\min \left\{d \in \mathbb{N} \mid\left(\boldsymbol{x}_{\mathfrak{v}_{i}}^{(i)} ; 0\right) \in \mathcal{X}_{1: d}\right\}\right]^{s}
$$

for some $s \in[0, \infty)$. Let us assume that our cost function $\$$ satisfies $\$(k)=\Theta\left(k^{s}\right)$. Then

$$
\operatorname{cost}(\mathcal{Q})=O\left(\operatorname{cost}^{\operatorname{var}}(\mathcal{Q})\right) .
$$

In the variable subspace sampling model, we define the $\varepsilon$-complexity $\operatorname{comp}^{\operatorname{var}}\left(\varepsilon ; \mathcal{H}_{\boldsymbol{\gamma}}\right)$ and the $N$ th minimal worst case error $e_{\gamma}^{\mathrm{var}}(N)$ by (17) and (19), where we simply substitute $\operatorname{cost}(\mathcal{Q})$ by $\operatorname{cost}^{\operatorname{var}}(\mathcal{Q})$. Analogously, we define (polynomial) tractability and the exponent of tractability $p^{\text {var }}$ in the variable subspace model. Due to (63) we have

$$
p^{*} \leq p^{\mathrm{var}}
$$

Thus our lower bounds for the exponent of tractability in the cost model from 22 . are also valid in the variable subspace sampling cost model. On the other hand, upper bounds for the exponent of tractability in the variable subspace sampling cost model are valid in the cost model from 22. But it is a priori not clear whether an upper bound in the cost model from [22] stays valid in the variable subspace sampling cost model.

This is indeed the case for our upper bounds achieved with the help of the multilevel algorithm. This is easy to see for lexicographically-ordered weights, finiteproduct and product weights. There we use the coordinate sets $\mathfrak{v}_{k}^{(2)}=\left[L_{k}\right], k=$ $1,2, \ldots$ for our multilevel algorithm $\mathcal{A}_{m}$ and therefore the cost of $\mathcal{A}_{m}$ is of the same order in both models; see also (38). For finite-intersection weights, this is not obvious and indeed an a-priori re-ordering of the coordinates might be necessary. So if we use a bijection $\zeta: \mathbb{N} \rightarrow \mathbb{N}$ satisfying $\zeta\left(\mathfrak{u}_{1}\right)=\left\{1, \ldots,\left|\mathfrak{u}_{1}\right|\right\}$ and

$$
\zeta\left(\mathfrak{u}_{j} \backslash \bigcup_{i=1}^{j-1} \mathfrak{u}_{i}\right)=\left\{\left|\bigcup_{i=1}^{j-1} \mathfrak{u}_{i}\right|+1, \ldots,\left|\bigcup_{i=1}^{j} \mathfrak{u}_{i}\right|\right\} \quad \text { for } j=2,3, \ldots,
$$

and permute the coordinates by using $\zeta$, then our algorithm $\mathcal{A}_{m}$ with coordinate sets $\tilde{\mathfrak{v}}_{k}^{(1)}:=\zeta\left(\mathfrak{v}_{k}^{(1)}\right)=\bigcup_{j \in\left[L_{k}\right]} \zeta\left(\mathfrak{u}_{j}\right), k=1,2, \ldots$, has also cost of the same order in both cost models ( since $\zeta\left(\mathfrak{v}_{k}^{(1)}\right)=\left[\left|\mathfrak{v}_{k}^{(1)}\right|\right]$ ). Observe that we can do the same a-priori re-ordering of coordinates for arbitrary weights and then use our algorithm $\mathcal{A}_{m}$ with the coordinate sets $\tilde{\mathfrak{v}}_{k}^{(1)}, k=1,2, \ldots$, defined as above. In this sense all upper bounds for the exponent of tractability achieved by our multilevel algorithm for the cost model from [22] will also hold for the variable subspace sampling cost model.

But the cost of the algorithm from [22, Thm. 5(a)] will, in general, differ reasonably in both models. Therefore we cannot claim that the upper bound in (20) holds 
also in the variable subspace sampling cost model. Thus, to hold in the variable subspace sampling model, the results in Theorems 3.12, 3.14, and 3.16 have to be modified accordingly, while the results from Theorem 4.2 hold without modification.

As already mentioned, the upper bound in Theorem 4.2 has been proved independently from our work in the recent paper [29] for the varying subspace sampling cost model (and thus, in particular, for the cost model from [22]). In [29] the authors consider a more general reproducing kernel Hilbert space setting than we consider in this paper, but study solely product weights. The multilevel algorithm proposed in [29] for infinite-dimensional integration over $\mathcal{H}_{\boldsymbol{\gamma}}$ with product weights $\gamma$ is similar to our multilevel algorithm; the main difference is that their algorithm uses quasi-Monte Carlo points proposed in [19], while our algorithm uses the points from Proposition 3.9. Although the multilevel approaches are similar, the analysis which establishes the upper bounds for $p^{\mathrm{var}}$ in [29] is quite different from ours and relies on auxiliary weights $\gamma^{\prime}$ with $\gamma_{\mathfrak{u}}^{\prime} \geq \gamma_{\mathfrak{u}}$ for all finite $\mathfrak{u} \subset \mathbb{N}$.

Remark 4.5. After the submission of this article, a new paper on infinite-dimensional integration on (quasi-)reproducing kernel Hilbert spaces was completed [33]. We briefly state the results relevant for the setting studied in our paper. The authors showed that in the cost model from [22 for cost functions $\$(k)=O\left(k^{s}\right)$ the bound on the exponent of tractability (59) holds also for product weights. This bound is sharp for $s \geq 1$ and $\$(k)=\Theta\left(k^{s}\right)$, as can be seen from (58). The proof uses changing dimension algorithms as in 22] (see Remark 4.3) and a refined analysis. The results from [33] cannot be transferred directly to the variable subspace sampling model, since the cost of these changing dimension algorithms will increase reasonably if one switches from the cost model in 22. to the variable subspace sampling model (see Remark 4.4). As explained in Remark 4.4, this is not the case for the multilevel algorithms presented in [29] and in our paper.

\section{ACKNOWLEDGMENT}

The author thanks Henryk Woźniakowski for motivating and helpful discussions, and Thomas Müller-Gronbach, Klaus Ritter, and Greg Wasilkowski for helpful comments. Furthermore, he acknowledges support from the German Science Foundation DFG under grants GN 91/3-1 and GN 91/4-1.

\section{REFERENCES}

[1] N. Aroszajn, Theory of reproducing kernels, Trans. Amer. Math. Soc. 68 (1950), 337-404. MR0051437 (14:479c)

[2] J. Baldeaux, Scrambled polynomial lattice rules for infinite-dimensional integration, preprint Math. Archive 2010, to appear in Monte Carlo and Quasi-Monte Carlo Methods 2010 .

[3] J. Creutzig, S. Dereich, T. Müller-Gronbach, K. Ritter, Infinite-dimensional quadrature and approximation of distributions, Found. Comput. Math. 9 (2009), 391-429. MR2519865 (2010h:65027)

[4] J. Dick, I. H. Sloan, X. Wang, H. Woźniakowski, Liberating the weights, J. Complexity 20 (2004), 593-623. MR2086942 (2005h:65008)

[5] J. Dick, I. H. Sloan, X. Wang, H. Woźniakowski, Good lattice rules in weighted Korobov spaces with general weights, Numer. Math. 103 (2006), 63-97. MR2207615(2006m:65014)

[6] B. Doerr, M. Gnewuch, M. Wahlström, Algorithmic construction of low-discrepancy point sets via dependent randomized rounding, J. Complexity 26 (2010), 490-507. MR2719644

[7] H. Faure, Discrépance de suites associées à un système de numèration (en dimension $s$ ), Acta Arith. 41 (1982), 337-351. MR677547 (84m:10050) 
[8] M. B. Giles, Multilevel Monte Carlo path simulation, Oper. Res. 56 (2008), 607-617. MR2436856 (2009g:65008)

[9] M. B. Giles, Improved multilevel Monte Carlo convergence using the Milstein scheme, in: A. Keller, S. Heinrich, H. Niederreiter (Eds.), Monte Carlo and Quasi-Monte Carlo Methods 2006, 343-358, Springer, Berlin-Heidelberg, 2008. MR2479233 (2010c:65007)

[10] M. B. Giles, B. J. Waterhouse, Multilevel quasi-Monte Carlo path simulation, Radon Ser. Comput. Appl. Math. 8 (2009), 165-181. MR2648461 (2011c:91261)

[11] M. Gnewuch, Weighted geometric discrepancies and numerical integration on reproducing kernel Hilbert spaces, J. Complexity 2011. (doi:10.1016/j.jco.2011.02.003)

[12] M. Gnewuch, H. Woźniakowski, Generalized tractability for multivariate problems, Part I: Linear tensor product problems and linear information, J. Complexity 23 (2007), 262-295. MR2314760 (2008d:65164)

[13] M. Gnewuch, H. Woźniakowski, Generalized Tractability for Linear Functionals, in: A. Keller, S. Heinrich, H. Niederreiter (Eds.), Monte Carlo and Quasi-Monte Carlo Methods 2006, 359-381, Springer, Berlin-Heidelberg, 2008. MR 2479234 (2010d:41040)

[14] J. H. Halton, On the efficiency of certain quasi-random sequences of points in evaluating multi-dimensional integrals, Numer. Math. 2 (1960), 84-90. MR0121961 (22:12688)

[15] S. Heinrich, Monte Carlo complexity of global solution of integral equations, J. Complexity 14 (1998), 151-175. MR1629093 (99g:65135)

[16] S. Heinrich, Multilevel Monte Carlo methods, in: S. Margenov, J. Wasniewski, P. Yalamov (Eds.), Large Scale Scientific Computing, LNiCS 2179, 58-67, Springer, Berlin, 2001.

[17] F. J. Hickernell, A generalized discrepancy and quadrature error bound, Math. Comp. 67 (1998), 299-322. MR.1433265(98c:65032)

[18] F. J. Hickernell, T. Müller-Gronbach, B. Niu, K. Ritter, Multi-level Monte Carlo algorithms for infinite-dimensional integration on $\mathbb{R}^{\mathbb{N}}$, J. Complexity 26 (2010), 229-254. MR2657363 (2011d:65008)

[19] F. J. Hickernell, I. H. Sloan, G. W. Wasilkowski, The strong tractability of multivariate integration using lattice rules, in: H. Niederreiter (Ed.), Monte Carlo and Quasi-Monte Carlo Methods 2002, Springer, Berlin, 2004. MR2076938

[20] F. J. Hickernell, X. Wang, The error bounds and tractability of quasi-Monte Carlo algorithms in infinite dimension, Math. Comp. 71 (2002), 1641-1661. MR.1933048(2003i:65009)

[21] S. Joe, Component by component construction of rank-1 lattice rules having $O\left(n^{-1}(\ln n)^{d}\right)$ star discrepancy, in: H. Niederreiter (Ed.), Monte Carlo and Quasi-Monte Carlo Methods 2002, 293-298, Springer, Berlin Heidelberg, 2004. MR2076940

[22] F. Y. Kuo, I. H. Sloan, G. W. Wasilkowski, H. Woźniakowski, Liberating the dimension, J. Complexity 26 (2010), 422-454. MR2719642

[23] F. Y. Kuo, I. H. Sloan, G. W. Wasilkowski, H. Woźniakowski, On decompositions of multivariate functions, Math. Comput. 79 (2010), 953-966. MR2600550 (2011a:41039)

[24] T. Müller-Gronbach, K. Ritter, Variable subspace sampling and multi-level algorithms, in: P. L'Ecuyer, A. B. Owen (Eds.), Monte Carlo and Quasi-Monte Carlo Methods 2008, 131-156, Springer, Heidelberg, 2009. MR2743892

[25] H. Niederreiter, Low-discrepancy and low-dispersion sequences, J. Number Theory 30 (1988), 51-70. MR960233 (89k:11064)

[26] H. Niederreiter, Random Number Generation and Quasi-Monte Carlo Methods, SIAM, Philadelphia, 1992. MR1172997 (93h:65008)

[27] H. Niederreiter, C. Xing, Low-discrepancy sequences and global function fields with many rational places, Finite Field Appl. 2 (1996), 241-273. MR1398076 (97h:11080)

[28] B. Niu, F. J. Hickernell, Monte Carlo simulation of stochastic integrals when the cost of function evaluations is dimension dependent, in: P. L'Ecuyer, A. B. Owen (Eds.), Monte Carlo and Quasi-Monte Carlo Methods 2008, 545-560, Springer, Heidelberg, 2009. MR.2743918

[29] B. Niu, F. J. Hickernell, T. Müller-Gronbach, K. Ritter, Deterministic multi-level algorithms for infinite-dimensional integration on $\mathbb{R}^{\mathbb{N}}$, J. Complexity 27 (2011), 331-351.

[30] E. Novak, H. Woźniakowski, $L_{2}$ discrepancy and multivariate integration, in: Analytic Number Theory: Essays in Honour of Klaus Roth, W. W. Chen, W. T. Gowers, H. Halberstam, W. M. Schmidt, R. C. Vaughan (Eds.), Cambridge University Press, 359-388, 2008. MR2508657 (2010d:11088) 
[31] E. Novak, H. Woźniakowski, Tractability of Multivariate Problems, Volume I, European Mathematical Society, Zürich, 2008. MR2455266 (2009m:46037)

[32] E. Novak, H. Woźniakowski, Tractability of Multivariate Problems, Volume II, European Mathematical Society, Zürich, 2010. MR2676032(2011h:46093)

[33] L. Plaskota, G. W. Wasilkowski, Tractability of infinite-dimensional integration in the worst case and randomized settings, J. Complexity 2011. (doi:10.1016/j.jco.2011.01.006)

[34] L. Plaskota, G. W. Wasilkowski, H. Woźniakowski, A new algorithm and worst case complexity for Feynman-Kac path integration, J. of Computational Physics 164 (2000), 335353. MR1792515 (2001i:65146)

[35] I. H. Sloan, X. Wang, H. Woźniakowski, Finite-order weights imply tractability of multivariate integration, J. Complexity 20 (2004), 46-74. MR2031558 (2004j:65034)

[36] I. H. Sloan, H. Woźniakowski, When are quasi-Monte Carlo algorithms efficient for high dimensional integrals?, J. Complexity 14 (1998), 1-33. MR.1617765 (99d:65384)

[37] I. M. Sobol', The distribution of points in a cube and the approximate evaluation of integrals, Zh. Vychisl. Mat. i Mat. Fiz. 7 (1967), 748-802. (Russian) MR0219238(36:2321)

[38] J. F. Traub, G. W. Wasilkowski, H. Woźniakowski, Information-Based Complexity, Academic Press, New York, 1988. MR958691 (90f:68085)

[39] G. W. Wasilkowski and H. Woźniakowski, On tractability of path integration, J. Math. Physics 37 (1996), 2071-2088. MR1380891 (97a:65010)

Department of Computer Science, Columbia University, 1214 Amsterdam Avenue, New York, New YORK 10027 